\title{
Patterns of Sources and Sinks in the Complex Ginzburg-Landau Equation with Zero Linear Dispersion*
}

\author{
Jonathan A. Sherratt ${ }^{\dagger}$, Matthew J. Smith ${ }^{\ddagger}$, and Jens D. M. Rademacher ${ }^{\S}$
}

\begin{abstract}
The complex Ginzburg-Landau equation with zero linear dispersion occurs in a wide variety of contexts as the modulation equation near the supercritical onset of a homogeneous oscillation. The analysis of its coherent structures is therefore of great interest. Its fundamental spatiotemporal pattern is wavetrains, which are spatially periodic solutions moving with constant speed (also known as periodic travelling waves and plane waves). In the past decade interfaces separating regions with different wavetrains have been studied in detail, as they occur both in simulations and in real experiments. The basic interface types are sources and sinks, distinguished by the signs of the opposing group velocities of the adjacent wavetrains. In this paper we study existence conditions for propagating patterns composed of sources and sinks. Our analysis is based on a formal asymptotic expansion in the limit of large source-sink separation and small speed of propagation. The main results concern the possible relative locations of sources and sinks in such a pattern. We show that sources and sinks are to leading order coupled only to their nearest neighbors, and that the separations of a source from its neighboring sinks, $L_{+}$and $L_{-}$say, satisfy a phase locking condition, whose leading order form is derived explicitly. Significantly this leading order phase locking condition is independent of the propagation speed. The solutions of the condition form a discrete infinite sequence of curves in the $L_{+}-L_{-}$plane.
\end{abstract}

Key words. source, sink, hole, shock, defect, coherent structure, Nozaki-Bekki hole, absolute stability, convective stability, pattern formation, $\lambda-\omega$ system, reaction-diffusion, partial differential equations

AMS subject classifications. 35Q56, 35K55, 35K57

DOI. $10.1137 / 090780961$

1. Introduction. Wavetrains are the fundamental solutions to spatially extended oscillatory systems. (Wavetrains are also referred to as plane waves and as periodic travelling waves in the literature.) In some contexts, an entire spatial domain is filled with a single wavetrain. However, in a number of areas of physics and chemistry, experiments reveal wavetrains arising not as single units spanning the whole domain, but rather as series of thinner bands separated by sharp interfaces. Most commonly these interfaces are alternating sequences of sources and sinks, defined by the condition that the group velocities of the two wavetrains are directed away from or towards the interface, respectively. Experimental systems in which sources and

\footnotetext{
* Received by the editors December 22, 2009; accepted for publication (in revised form) by D. Barkley May 5, 2010; published electronically July 27, 2010.

http://www.siam.org/journals/siads/9-3/78096.html

${ }^{\dagger}$ Corresponding author. Department of Mathematics and Maxwell Institute for Mathematical Sciences, HeriotWatt University, Edinburgh EH14 4AS, UK (jas@ma.hw.ac.uk). This author was supported in part by a Research Fellowship from the Leverhulme Trust.

${ }^{\ddagger}$ Microsoft Research, 7 J. J. Thompson Avenue, Cambridge CB3 0FB, UK (Matthew.Smith@microsoft.com). This author was supported by Microsoft Research Cambridge (MSRC).

${ }^{\S}$ Centrum Wiskunde en Informatica, Science Park 123, 1098 XG Amsterdam, The Netherlands (Jens. Rademacher@cwi.nl). This author was supported by the NWO program NDSN+.
} 
sinks have been observed include chemical reactions [37], electrochemical systems [39], heated wire convection [1,35, 36], binary fluid convection [26, 24], convection waves generated by heating at a boundary [7], and the "printer's instability," in which the thin gap between two rotating acentric cylinders is filled with oil $[17,16]$. Sources and sinks are believed to play an important role in the development of spatiotemporal chaos; for example, the repeated creation and destruction of sources and sinks is a hallmark of "defect chaos" [57, 2, 56].

Sources and sinks have been studied theoretically in a variety of equations, including the cubic-quintic complex Ginzburg-Landau equation [60, 10], coupled complex Ginzburg-Landau equations [59, 31], and reaction-diffusion systems [46]. However, it is in the (cubic) complex Ginzburg-Landau equation (CGLE) that the most comprehensive work has been done (see [2]). In this context, sources are often known as holes because they have the form of a local dip in amplitude, while sinks are often known as shocks. Nozaki and Bekki [34] showed that the CGLE has a one-parameter family of hole solutions; the family is typically parameterized using the hole velocity or the amplitude of one of the asymptotic wavetrains. The subsequent literature on Nozaki-Bekki holes is extensive: for reviews, see Stiller et al. [55], Lega [29], and Aranson and Kramer [2, section III.B]. From a strict mathematical point of view, Nozaki-Bekki holes are isolated objects, separating two semi-infinite expanses of wavetrains. In practice, both in numerical simulations of the CGLE and in experiments, one usually sees patterns involving a series of sources that resemble Nozaki-Bekki holes, separated by sinks. The sources and sinks can be stationary, can move together as a coherent unit, or can move with different velocities. Patterns of these types have been very widely reported; for a variety of examples, see Chaté [8]. However, there are almost no results on the possible forms of source-sink patterns. In this paper, we study these patterns in detail for the case of the CGLE with zero linear dispersion:

$$
\begin{aligned}
& u_{t}=u_{x x}+\left(1-r^{2}\right) u+c r^{2} v, \\
& v_{t}=v_{x x}+\left(1-r^{2}\right) v-c r^{2} u,
\end{aligned}
$$

where $r=\left(u^{2}+v^{2}\right)^{1 / 2}$ and the parameter $c>0$ is the coefficient of nonlinear dispersion. Note that writing $A=u+\mathrm{i} v$ transforms (1) into the more usual CGLE form

$$
A_{t}=A_{x x}+A-(1+\mathrm{i} c)|A|^{2} A .
$$

These equations are a reaction-diffusion system, of a type often referred to as " $\lambda-\omega$ equations" (Kopell and Howard [27]). The CGLE appears in broad generality as the modulation equation near the onset of Hopf-type instabilities [2]. The case of zero linear dispersion occurs, for instance, at a standard supercritical Hopf bifurcation of a homogeneous equilibrium state in a reaction-diffusion equation with equal diffusivities. We focus on this case because the resulting mathematical simplifications make it possible to obtain analytic results.

Simulation-based studies of oscillatory reaction-diffusion equations have shown that wavetrains often arise when initial or boundary conditions are inconsistent with spatially uniform oscillations $[15,25,52]$. A typical scenario is a Dirichlet boundary condition at one edge of a large bounded domain, and our work on source-sink patterns began with an investigation of the solutions of (1) on $0<x<L$ with $L$ large, subject to $u=v=0$ at $x=0$ and $u_{x}=v_{x}=0$ at $x=L$. The results of this investigation have been presented previously (Smith, Rademacher, and Sherratt [53]), and we give only a brief summary of the salient 
points. When $c$ is sufficiently small, the long-term solution away from the boundaries consists of a wavetrain of amplitude

$$
R^{*}=\left(\frac{1}{2}\left[1+\sqrt{1+\frac{8}{9} c^{2}}\right]\right)^{-1 / 2} .
$$

This wavetrain is stable when $c<1.110468$ [27] and absolutely stable if $c<1.576465$ [53]. (Here and throughout the paper we use the term "(un)stable" to mean (un)stable spectrum of the linearization about the wavetrain.) When $c$ is above the absolute stability threshold, the long-term behavior in our simulations is spatiotemporal disorder. However, for $c$ between the two threshold values, so that the wavetrain of amplitude $R^{*}$ is convectively unstable, one sees bands of the wavetrain solution, separated by sources and sinks; typical examples are illustrated in Figure 1. Note that stability of the asymptotic wavetrains is not a prerequisite for stability of source-sink patterns. If the asymptotic wavetrains are convectively unstable, then any unstable linear mode propagates as it grows (see, for example, Sandstede and Scheel [46] and the references therein). Such a growing mode therefore travels to the nearest sink, where it is absorbed; thus the solution as a whole is stable in a suitable function space. This situation is reminiscent of the stability of pulses in reaction-diffusion systems when the two asymptotic states are convectively stable (Sandstede and Scheel [48], Nii [33], Romeo and Jones [42]). However, if the asymptotic wavetrain is absolutely unstable, there is a stationary unstable linear mode, rendering a source-sink pattern pointwise unstable. We discuss the stability of source-sink patterns in more detail in section 6.

In an extensive program of numerical simulations of the type shown in Figure 1, we were struck by the continual repetition (for any given $c$ ) of a few relatively small values of the separation distance between sources and neighboring sinks. Larger separation distances were not repeated in the same way, but in section 5.2 we show that this is due to numerical inaccuracies. The observation of repeated separation distances motivated our study of source-sink separations, which forms the subject of this paper. We began with a numerical investigation. Working on a domain of given size, we constructed initial conditions with the form of a source located at an arbitrary (and controllable) location between two sinks, with the objective of tracking any movement of the source from its initial location. This simple idea is in fact rather difficult to implement. One important issue is boundary conditions. We cannot use periodic boundary conditions either for $u$ and $v$ or for $r$ and $\theta$ because these would immediately impose a constraint on the possible source-sink separations. Therefore we used instead $u_{x}=v_{x}=0$ at both boundaries. This condition is compatible with the center of a sink, so that we need only specify and track the location of the source in the interior of the domain. However, $u_{x}=v_{x}=0$ is also satisfied by the (stable) spatially uniform oscillation $u=\sin (c t), v=\cos (c t)$, and the basic difficulty with the study is that this uniform oscillation has a very large basin of attraction. This led to the failure of our initial approach, in which we constructed an initial condition by gluing together an isolated source (which has a known analytical form; see Newell [32], Nozaki and Bekki [34], Sherratt [51]), an isolated sink (which can easily be calculated numerically to very high accuracy), and sections of asymptotic wavetrain. This initial condition is, at least superficially, extremely similar to a coupled sink-source-sink triple, but it evolves to the spatially uniform oscillation.

Copyright (c) by SIAM. Unauthorized reproduction of this article is prohibited. 

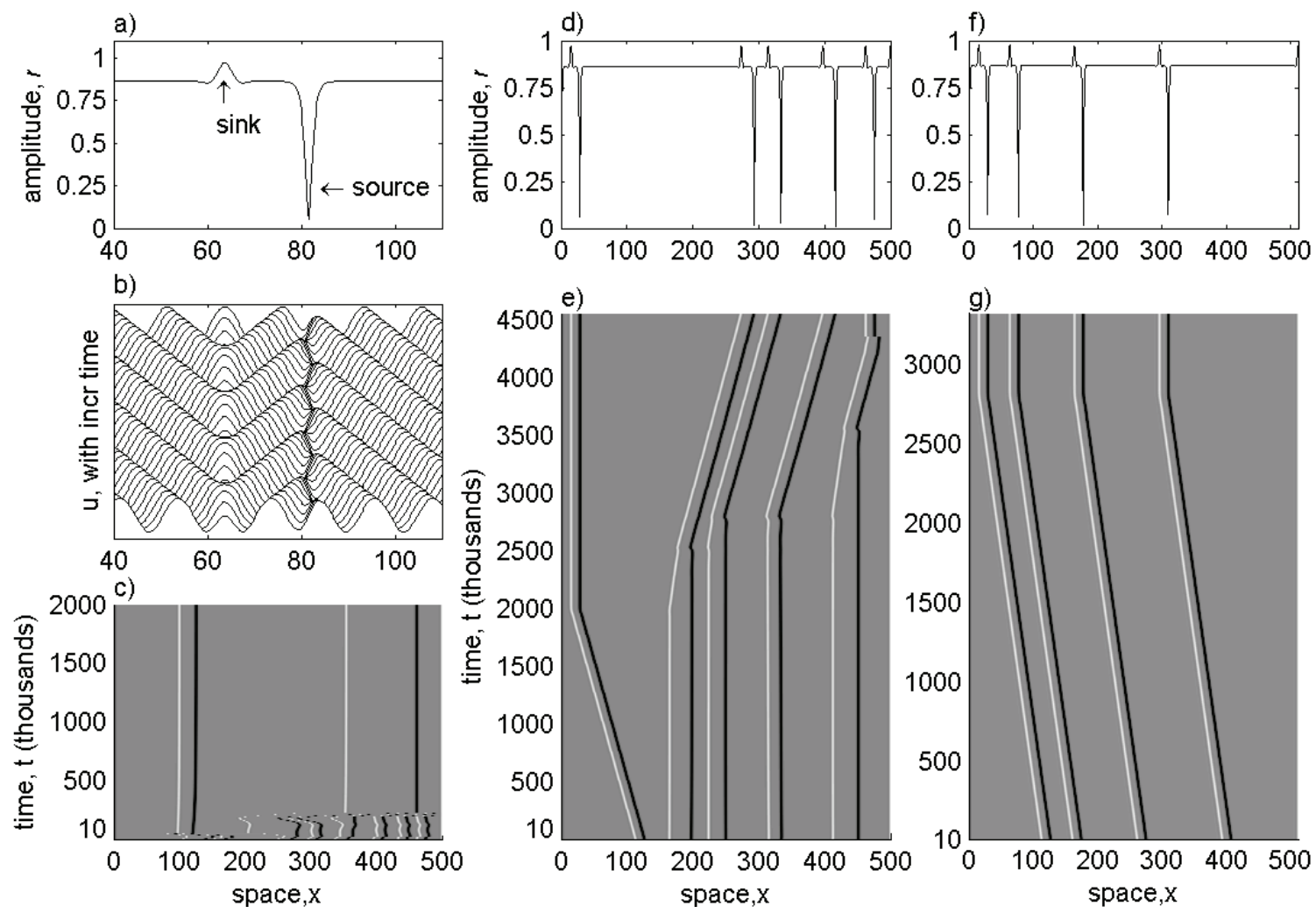

Figure 1. Repeating patterns of sources and sinks predicted by (1), with boundary conditions $u=v=0$ at $x=0$ and $u_{x}=v_{x}=0$ at $x=500$. These conditions force a boundary source at $x=0$ and a boundary sink at the right-hand boundary $x=500$. The nonlinear dispersion parameter $c=1.45$ in (a)-(e), and $c=1.4$ in $(\mathrm{f})$ and $(\mathrm{g})$. For these values, the asymptotic wavetrain of the (stationary) boundary source is convectively unstable (i.e., unstable but absolutely stable), and patterns of sources and sinks develop in the interior of the domain [53]. Initial conditions for $u$ and $v$ were drawn from uniformly distributed random numbers between 1 and 0. (a) A close-up of a source-sink solution at time $t=1000$, where amplitude $r=\left(u^{2}+v^{2}\right)^{1 / 2}$. (b) The spatiotemporal dynamics of $u$ for the same simulation between $t=975$ and $t=1000$. (c), (e), and (g) The long-term spatiotemporal dynamics of $r$, with darker shading indicating smaller $r$ and $0<r<1$. Thus sources and sinks appear as black and white lines, respectively. (d) and (f) The solutions for $r$ at the final time points in $(\mathrm{e})$ and $(\mathrm{g})$, respectively. The simulations in (c) and (e) differ only in the seed that was used to generate the random initial conditions. The equations were solved numerically using a semi-implicit Crank-Nicolson method, with $\delta x=0.2$ and $\delta t=0.001$. For $c=1.0$, say, the computed (stable) asymptotic wavetrain amplitude is then accurate to $0.012 \%$.

Instead, we took initial conditions from the results of our Dirichlet boundary condition simulations. We extracted the portion of one of these solutions between two adjacent sinks. We then relocated the intermediate source by altering the length of the regions, away from the source and (boundary) sinks, in which the solution is approximately on the asymptotic wavetrain. Appropriate phase shifts are required in the various parts of the solution, to ensure continuity of $u$ and $v$. When the initial change in source location was small, we found that the source returned to its original position; however, sufficiently large translations cause the source to move to a new location (Figure 2).

Copyright (c) by SIAM. Unauthorized reproduction of this article is prohibited. 

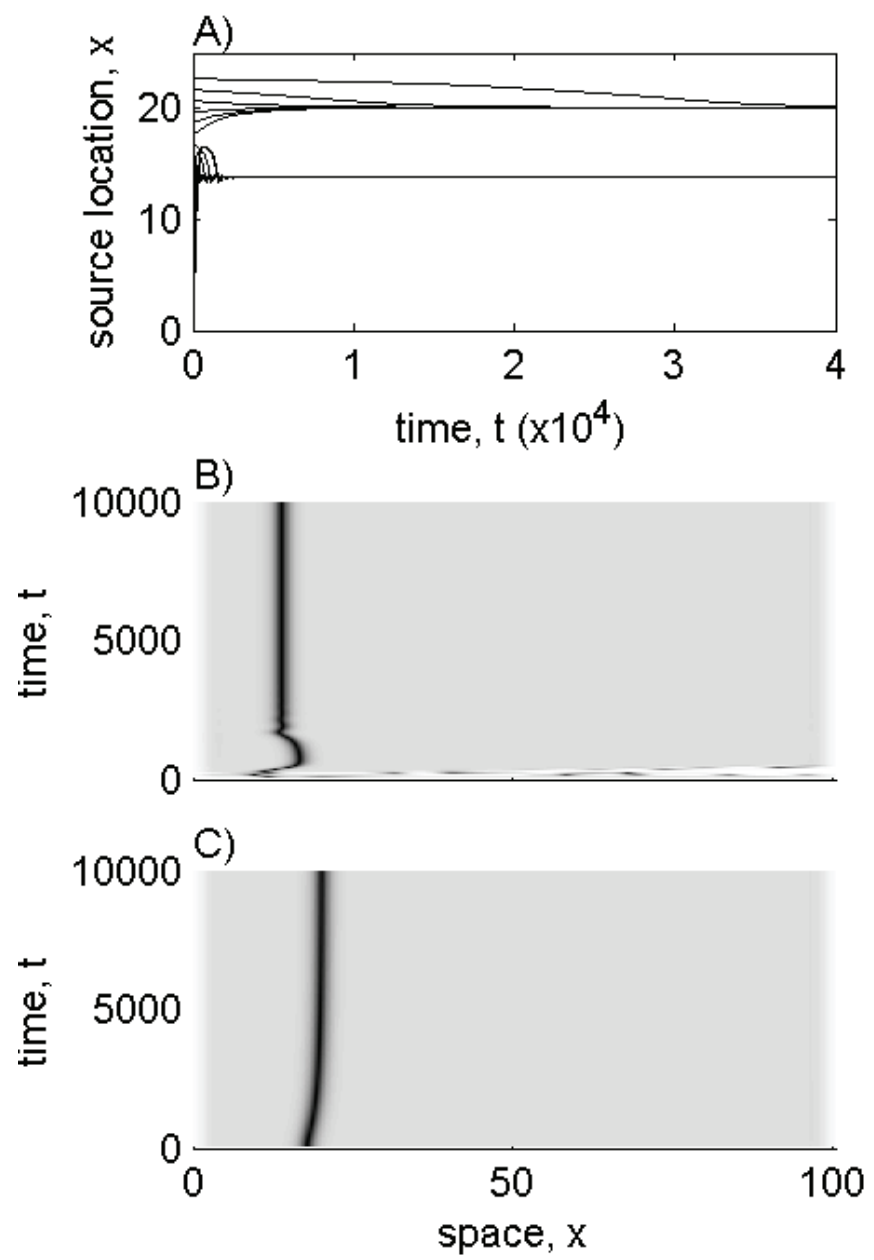

Figure 2. Results of numerical experiments on the translation of the source in a sink-source-sink triple. We extracted such a triple from a numerical solution of (1) subject to zero Dirichlet boundary conditions on a large domain; solutions of this type are illustrated in Figure 1. The sink-source-sink triple that we chose had source-sink separations of 13.8 and 86.6. We then relocated the source as described in the main text, giving triples with source-sink separations of $13.8+n \delta x$ and $86.6-n \delta x$, with $n=-4,-3, \ldots,+9$; here $\delta x=0.2$ is the spacing of our numerical grid. We used these triples as initial conditions in numerical solutions of (1) subject to $u_{x}=v_{x}=0$ at $x=0$ and $x=100.4$; these end conditions are compatible with sinks. (a) The time courses of source location; (b), (c) the spatiotemporal dynamics of $r$ for $n=-4$ and +4 , with darker shading indicating smaller $r(0<r<1)$. For $-4 \leq n \leq 3$, the source returns to its original location, but for $4 \leq n \leq 9$, it moves to a new location with source-sink separations of 20.0 and 80.4. The equations were solved numerically using a semi-implicit Crank-Nicolson method, with $\delta x=0.2$ and $\delta t=0.001$. The parameter $c=1.4$.

These results suggest that the separation of sources and sinks may be restricted to a discrete set of possible values. Our goal in the remainder of this paper is to investigate sourcesink separation in detail, and to calculate the possible separation distances. We consider patterns in which the propagation velocity is small and the separation between each source and sink is large. Our results apply to leading order as the velocity tends to zero and the

Copyright (c) by SIAM. Unauthorized reproduction of this article is prohibited. 
separations tend to infinity (precise statements are given later). Our key findings from a formal leading order asymptotic matching are, roughly speaking, as follows:

1. For a single source-sink pair moving with any fixed small velocity, there is a discrete infinite sequence of possible separations with constant increments, to leading order.

2. For patterns with multiple sources and sinks moving with any fixed small velocity, the separations $L_{+}$and $L_{-}$between each source and its neighboring sinks are constrained to lie on one of a discrete infinite sequence of curves in the $L_{+}-L_{-}$plane. The translation between any two consecutive curves in the sequence is the same, and as $L_{+}$(say) $\rightarrow \infty$, the asymptotic values of $L_{-}$tend to the separations in 1 .

3 . In both 1 and 2 , we derive formulae for the possible separations, valid to leading order. The key feature that generates these behaviors is that, for sinks, the solution amplitude has an oscillatory decay to its constant value in the asymptotic wavetrain. Since we are considering small propagation velocities, this is a simple consequence of the asymptotic wavetrain having a pair of complex spatial eigenvalues; these complex eigenvalues emerge below the onset of convective instability and are present throughout the unstable regime. The condition that determines the possible separations $L_{ \pm}$corresponds to phase matching for this oscillatory decay.

Our work is distinct from the one previous paper we are aware of that addresses the general issue of source-sink separations (Popp et al. [40]). Those authors obtain results on the separation of sources and sinks in the CGLE perturbed by a small quintic term. But crucially their results (which are qualitatively different from ours) apply only when the coefficient of the quintic term, though small, is large compared to the negative exponential of the separation (multiplied by a particular scaling factor). Consequently, at large separations, their results apply fundamentally to the cubic-quintic CGLE.

2. Formulation of the problem. It is most convenient to study (1) in terms of amplitude $r$ and phase $\theta=\tan ^{-1}(v / u)$, which satisfy

$$
\begin{aligned}
& r_{t}=r_{x x}-r \theta_{x}^{2}+r\left(1-r^{2}\right), \\
& \theta_{t}=\theta_{x x}+\frac{2 r_{x} \theta_{x}}{r}-c r^{2} .
\end{aligned}
$$

The wavetrain family is $r=R, \theta=\theta_{0} \pm \sqrt{1-R^{2}} x-c R^{2} t$ [27]; here $\theta_{0}$ is an arbitrary constant, and the amplitude $R$ is the most natural parameter for the family, with $0 \leq R \leq 1$. The phase velocity of wavetrains is $\pm c R^{2} / \sqrt{1-R^{2}}$, and their group velocity is $\mp c \sqrt{1-R^{2}}$; since these have opposite signs, wavetrains propagate towards sources and away from sinks. Note that these directions can be reversed via the gauge invariance of the CGLE.

The source-sink patterns that we consider have amplitude $r$ and phase gradient $\theta_{x}$ changing with constant shape and (slow) speed (see Figure 1, for example). Therefore the solution ansatz

$$
r(x, t)=\widehat{r}(z), \quad \theta_{x}(x, t)=\widehat{\psi}(z), \quad z=x-s t
$$

is appropriate. Here $s$ is the speed of source/sink movement, which can take either sign or be zero. Then $\theta(x, t)=\int^{z=x-s t} \widehat{\psi}(z) d z+\widehat{\theta}(t)$ for some function $\widehat{\theta}($.$) , and substitution into (3)$ 


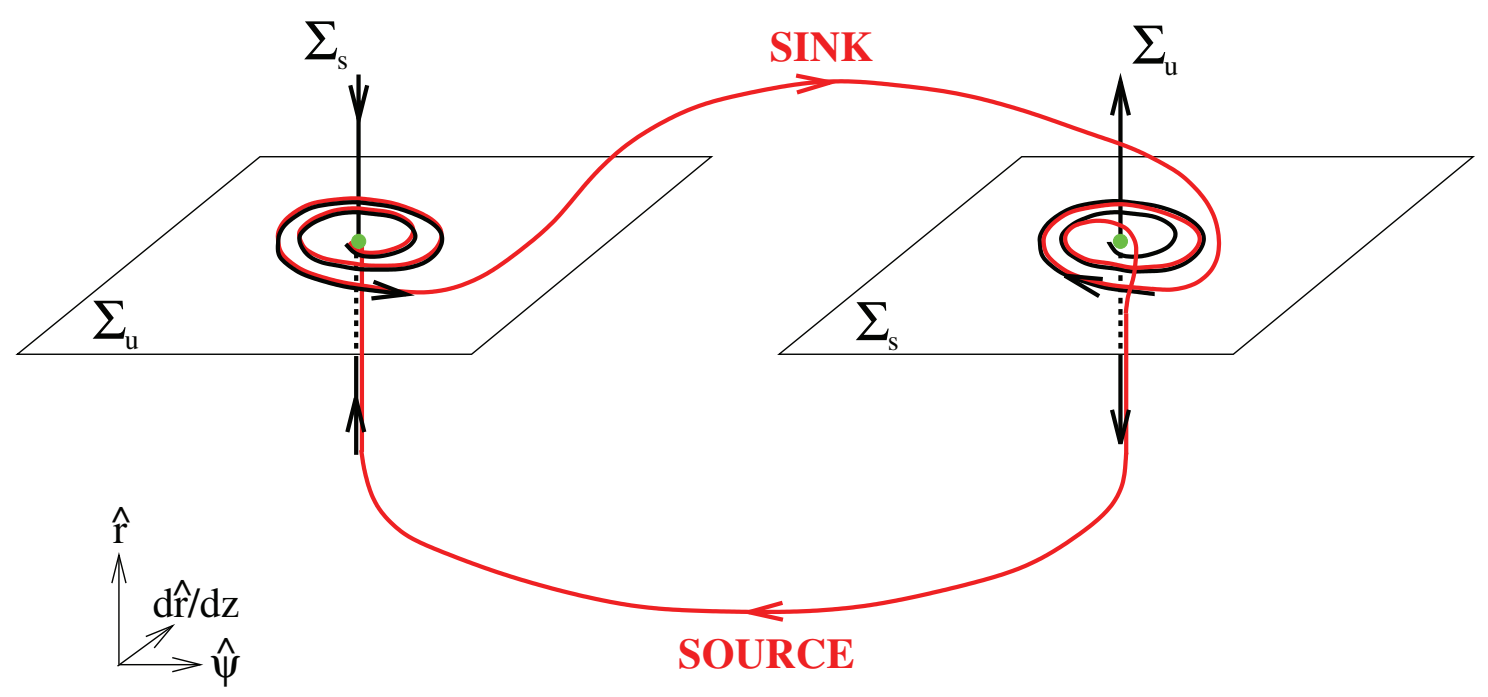

Figure 3. A schematic illustration of the solution trajectory of (5) corresponding to an isolated source-sink pair. The green dots denote the asymptotic wavetrains. Their unstable and stable manifolds $\left(\Sigma_{u}\right.$ and $\left.\Sigma_{s}\right)$, and the corresponding flows, are shown in black. The red curve denotes the solution trajectory. Note that this trajectory is close to a heteroclinic cycle, which is the organizing center for all source-sink patterns. This sketch can be viewed in parallel with Figure 8 below, which shows numerically calculated profiles of $\widehat{r}(z)$ and $\widehat{\psi}(z)$ for an isolated source-sink pair.

gives

$$
\begin{aligned}
& \frac{d^{2} \widehat{r}}{d z^{2}}+s \frac{d \widehat{r}}{d z}+\widehat{r}\left(1-\widehat{r}^{2}-\widehat{\psi}^{2}\right)=0 \\
& \frac{d \widehat{\psi}}{d z}+s \widehat{\psi}-c \widehat{r}^{2}+2 \frac{\widehat{\psi} d \widehat{r} / d z}{\widehat{r}}=\frac{d \widehat{\theta}(t)}{d t} .
\end{aligned}
$$

It follows from $(5 \mathrm{~b})$ that $d \widehat{\theta}(t) / d t$ is a constant, independent of $z$ and $t$, and its value is henceforth denoted by $K$. We require that $\widehat{r}$ and $\widehat{\psi}$ approach constant values in between the sources and sinks: these are the amplitude and phase gradient of the asymptotic wavetrains. Figure 3 illustrates the solution trajectory of (5) corresponding to an isolated source-sink pair. Adopting a notational convention that will be continued throughout the paper, we denote by $R^{+}$(respectively, $R^{-}$) the amplitude of the asymptotic wavetrain to the right (respectively, left) of a source. The corresponding values of $\widehat{\psi}$ are then $\mp\left(1-R^{ \pm 2}\right)^{1 / 2}$, and (5) implies that

$$
\mp s\left(1-R^{ \pm 2}\right)^{1 / 2}-c R^{ \pm 2}=K .
$$

When a source is widely separated from neighboring $\operatorname{sink}(\mathrm{s})$, its form is similar to a Nozaki-Bekki hole [34]. When additionally the propagation velocity is small, it is the (unique) stationary hole solution that is relevant. This has a particularly simple analytical form:

$$
\left(\begin{array}{l}
u \\
v
\end{array}\right)=R^{*} \tanh (x / \sqrt{2})\left(\begin{array}{c}
\cos \\
\sin
\end{array}\right)\left[-c R^{* 2} t \mp \operatorname{sign}(c) \sqrt{2\left(1-R^{* 2}\right)} \log \cosh (x / \sqrt{2})\right],
$$


where $R^{*}$ is given by $(2)[32,34,51]$. Our combined assumptions of large source-sink separations and slow propagation speed mean that $R^{+}$and $R^{-}$will both be close to $R^{*}$. It is the small size of $R^{ \pm}-R^{*}$ that constitutes the formal basis for the perturbation theory calculation that we will present in section 3 . We write

$$
\begin{aligned}
s & =\epsilon s_{1}+\epsilon^{2} s_{2}+\cdots, \\
K & =K_{0}+\epsilon K_{1}+\epsilon^{2} K_{2}+\cdots, \\
R^{ \pm} & =R^{*}+\epsilon R_{1}^{ \pm}+\epsilon^{2} R_{2}^{ \pm}+\cdots,
\end{aligned}
$$

where $\epsilon \ll 1$. In order for the $R_{i}^{ \pm}$'s to be specified uniquely, a normalization condition is required, and we set

$$
\epsilon=c R^{* 2}\left(2-\frac{R^{+}+R^{-}}{R^{*}}\right)
$$

which gives some algebraic simplification in the subsequent calculations. Then $R_{1}^{+}+R_{1}^{-}=$ $-1 /\left(c R^{*}\right)$, and $R_{i}^{+}+R_{i}^{-}=0$ for $i \geq 2$. Note that $\epsilon$ can take either sign. The basic objective of section 3 is to derive the leading order relationship between $\epsilon$ and the source-sink separation.

To leading order, (6) and (8) imply

$$
K_{0}=-c R^{* 2}
$$

Moreover, (6) has one real root for each of $R^{ \pm}$when $\epsilon$ is sufficiently small. Substituting (9) into (6) and equating coefficients of $\epsilon$ implies that $K_{1}=1$ and

$$
s_{1}=c R^{*}\left(R_{1}^{-}-R_{1}^{+}\right) / \sqrt{1-R^{* 2}} .
$$

This proportionality between the propagation speed and the difference in asymptotic wavetrain amplitudes is well known (e.g., Alvarez, van Hecke, and van Saarloos [1], Sherratt [51], Sandstede and Scheel [46, sections 6.2-6.3]). We emphasize that there is a 1-1 correspondence between $\left(\epsilon, s_{1}\right)$ and $\left(R_{1}^{+}, R_{1}^{-}\right)$: inverting (9) and (11) implies

$$
R_{1}^{ \pm}=-\frac{\epsilon}{2 c R^{*}} \mp \frac{s_{1} \sqrt{1-R^{* 2}}}{2 c R^{* 2}} .
$$

3. Analytical investigation of sink-source-sink patterns. Having established the underlying parameterization of the problem, we can proceed with our calculation, in which we regard (5) as a perturbation theory problem in the small parameter $\epsilon$, treating the source-sink separations as functions of $\epsilon$. Throughout, we use $\epsilon$ and $s_{1}$ as parameters, rather than $R_{1}^{ \pm}$. Also, we use $R^{*}$ rather than $c$ as the physical parameter; the two are equivalent via (2), which inverts to give

$$
c=3 \sqrt{1-R^{* 2}} /\left(\sqrt{2} R^{* 2}\right)
$$

(using our assumption that $c>0$ ). We write

$$
\begin{aligned}
& \widehat{r}=\widehat{r}_{0}(z)+\epsilon \widehat{r}_{1}(z)+\epsilon^{2} \widehat{r}_{2}(z)+\cdots, \\
& \widehat{\psi}=\widehat{\psi}_{0}(z)+\epsilon \widehat{\psi}_{1}(z)+\epsilon^{2} \widehat{\psi}_{2}(z)+\cdots .
\end{aligned}
$$

Copyright (c) by SIAM. Unauthorized reproduction of this article is prohibited. 


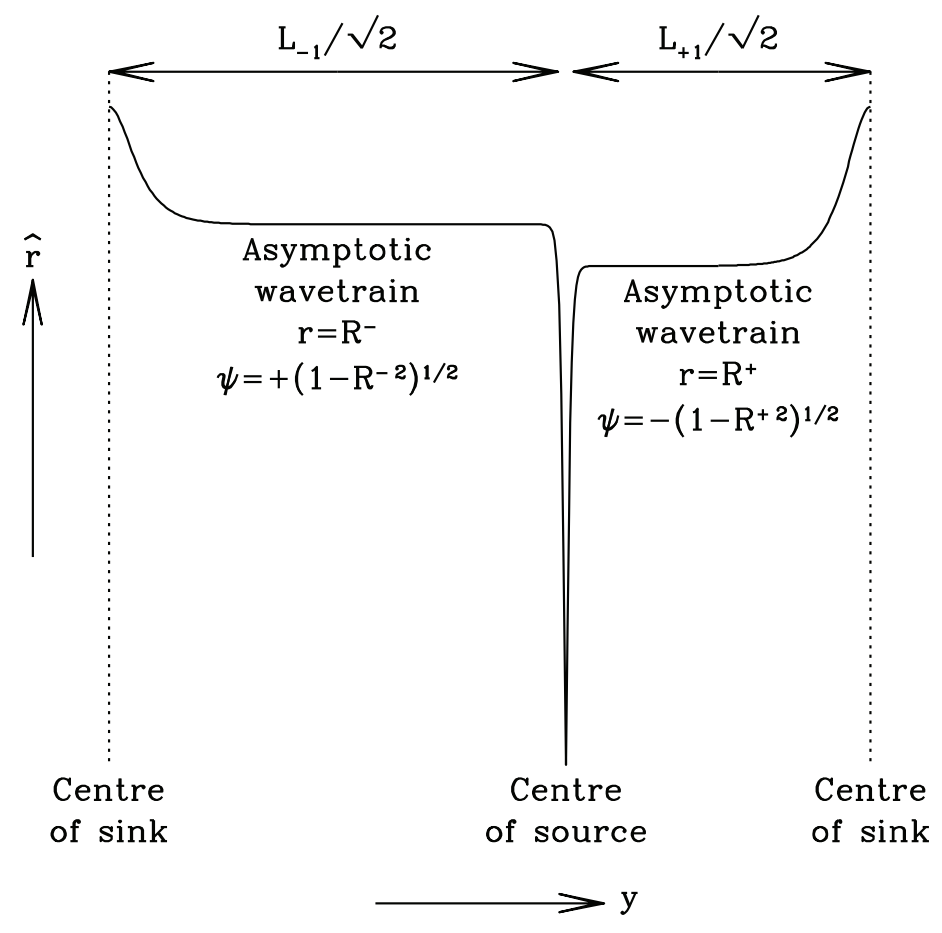

Figure 4. A schematic illustration of the problem set-up. The rescaled coordinate $y$ is related to $z=x-$ st via $y=z / \sqrt{2}$.

Substituting these expansions into (5); using (6), (8), and (10); and equating terms independent of $\epsilon$ gives

$$
\begin{aligned}
\frac{d^{2} \widehat{r}_{0}}{d z^{2}}+\widehat{r}_{0}\left(1-\widehat{r}_{0}^{2}-\widehat{\psi}_{0}^{2}\right) & =0 \\
\frac{d \widehat{\psi}_{0}}{d z}+2 \frac{\widehat{\psi}_{0} d \widehat{r}_{0} / d z}{\widehat{r}_{0}}+\left(\frac{3}{\sqrt{2}}\right)\left(1-R^{* 2}\right)^{1 / 2}\left(1-\frac{\widehat{r}_{0}^{2}}{R^{* 2}}\right) & =0 .
\end{aligned}
$$

We will consider the behavior on the two sides of a source centered at $z=0$, with neighboring sinks centered at $z=\xi L_{\xi}(\epsilon)$. Here $\xi= \pm 1$ and $L_{\xi}>0$ with $L_{\xi}(\epsilon) \rightarrow \infty$ as $\epsilon \rightarrow 0$; recall that the asymptotic wavetrain amplitudes are $R^{+}$for $z>0$ and $R^{-}$for $z<0$. The problem set-up is illustrated schematically in Figure 4 . In the stationary case $\left(s_{1}=0\right)$, the solution is the same for $\xi=+1$ and $\xi=-1$, but for the moving case $\left(s_{1} \neq 0\right)$, this formulation avoids separate calculations for the cases of a source upstream or downstream of a sink. To formally specify the location of the origin of $z$, we impose the condition that $\widehat{r}$ has a local minimum at $z=0$. To leading order, the wavetrain band in between the source and sink (specifically at $\left.1 \ll z \ll L_{\xi}(\epsilon)\right)$ has $\widehat{r}=R^{*}$ and $\widehat{\psi}=-\xi \sqrt{1-R^{* 2}}$. (Recall that $\widehat{\psi}<0$ for a wavetrain moving in the negative $x$ direction - and thus with positive group velocity - and vice versa.) Linearizing (15) about this wavetrain solution gives a third order linear ODE system whose characteristic values are calculated as $-\xi \sqrt{2}$ and $+\xi\left(1 \pm i \sqrt{11-12 R^{* 2}}\right) / \sqrt{2}$; note that we are concerned only with values of $c$ for which the (leading order) wavetrain is 
unstable, i.e., $c>1.110468 \Rightarrow R^{*}<0.903914$ (using (2)), so that $11-12 R^{* 2}>0$. Therefore the leading order source solution (centered at $z=0$ ) decays towards the asymptotic wavetrain at a rate that is twice that for the sink (centered at $z=\xi L_{\xi}(\epsilon)$ ). Crucially, this implies that the dominant interaction between the source and sink is via the effect of the sink solution on the source, with the reverse interaction being of lower order. Therefore in the remainder of the calculation we focus on the first order correction to the source, and its matching to the leading order sink solution.

3.1. Perturbing the source. A detailed study is possible because there is an exact solution for the leading order (isolated) source (see (7)), namely

$$
\begin{aligned}
\widehat{r}_{0} & =\xi R^{*} \tanh y, \\
\widehat{\psi}_{0} & =-\sqrt{1-R^{* 2}} \tanh y,
\end{aligned}
$$

where $R^{*}$ is defined in (2) $[32,34,51]$. Here and henceforth we write $y=z / \sqrt{2}$ for notational simplicity; note that $y$ takes positive values when $\xi=+1$ and negative values when $\xi=-1$. With these formulae, substituting (14) into (5) (rewritten in terms of $y$ rather than $z$ ) and equating coefficients of $\epsilon$ gives

$$
\begin{aligned}
d^{2} \widehat{r}_{1} / d y^{2}+\xi s_{1} R^{*} \sqrt{2} \operatorname{sech}^{2} y+ & 2 \widehat{r}_{1}\left[1-\left(1+2 R^{* 2}\right) \tanh ^{2} y\right] \\
& +4 \xi R^{*} \sqrt{1-R^{* 2}} \tanh ^{2} y \widehat{\psi}_{1}=0,
\end{aligned}
$$

Differentiating (17a) with respect to $y$ enables elimination of $\widehat{\psi}_{1}$, giving a third order ODE for $\widehat{r}_{1}$ :

$$
\begin{aligned}
d^{3} \widehat{r}_{1} / d y^{3} & +2\left(d \widehat{r}_{1} / d y\right)\left[1-3\left(2 R^{* 2}-1\right) \tanh ^{2} y\right] \\
& -12 \widehat{r}_{1}\left[\tanh y \operatorname{sech}^{2} y-2\left(1-R^{* 2}\right) \tanh ^{3} y\right]=g(y)
\end{aligned}
$$

where

$$
g(y)=2 \sqrt{2} \xi R^{*} \tanh y\left[s_{1}\left\{1+\left(2 R^{* 2}-3\right) \tanh ^{2} y\right\}-2 \sqrt{\left(1-R^{* 2}\right)} \tanh y\right] .
$$

This equation can be solved exactly via a substitution that reduces the corresponding homogeneous equation to a hypergeometric equation. This approach was used previously by Sherratt [50] and Smith, Sherratt, and Armstrong [54], who derived asymptotic expansions for solutions satisfying boundary conditions close to a one-dimensional zero Dirichlet condition. We omit details of the solution method for brevity, referring the reader to either of these previous papers for details. The result is the following general solution of (18):

Copyright $\odot$ by SIAM. Unauthorized reproduction of this article is prohibited. 


$$
\begin{aligned}
\widehat{r}_{1}(y)= & \frac{\operatorname{sech}^{2} y}{W}\left[\int_{y_{1}=0}^{y_{1}=y} Y^{-}\left(y_{1}\right) \int_{y_{2}=y_{1}}^{y_{2}=\xi \infty} \frac{Y^{+}\left(y_{2}\right) g\left(y_{2}\right)}{\cosh ^{4} y_{2}} d y_{2} d y_{1}\right. \\
& \left.\quad-\int_{y_{1}=0}^{y_{1}=y} Y^{+}\left(y_{1}\right) \int_{y_{2}=y_{1}}^{y_{2}=\xi \infty} \frac{Y^{-}\left(y_{2}\right) g\left(y_{2}\right)}{\cosh ^{4} y_{2}} d y_{2} d y_{1}\right] \\
& +C_{1}^{\xi} \operatorname{sech}^{2} y \int_{y_{1}=0}^{y_{1}=y} Y^{+}\left(y_{1}\right) d y_{1}+C_{2}^{\xi} \operatorname{sech}^{2} y \int_{y_{1}=0}^{y_{1}=y} Y^{-}\left(y_{1}\right) d y_{1} \\
& +C_{3}^{\xi} \operatorname{sech}^{2} y
\end{aligned}
$$

where

$$
\begin{aligned}
Y^{ \pm}(y) & =\operatorname{Re}\left[\operatorname{sech}^{-3+i \delta} y F\left(\alpha, \beta, \gamma, \frac{1 \pm \tanh y}{2}\right)\right], \\
\alpha, \beta & =\frac{1}{2}+i\left(\delta \pm \sqrt{\delta^{2}+\frac{3}{4}}\right), \\
\gamma & =1+i \delta \\
\delta & =\sqrt{11-12 R^{* 2}} \\
W & =4 \pi \operatorname{Re}\left[\frac{\Gamma(\alpha \beta)}{\Gamma\left(\frac{1+\alpha}{2}\right) \Gamma\left(\frac{1+\beta}{2}\right)}\right] \cdot \operatorname{Re}\left[\frac{\Gamma(\alpha \beta)}{\Gamma\left(\frac{\alpha}{2}\right) \Gamma\left(\frac{\beta}{2}\right)}\right] .
\end{aligned}
$$

Here $F$ is the hypergeometric function, and $C_{j}^{\xi}(j=1,2,3)$ are constants of integration.

This solution contains seven undetermined constants: $C_{1}^{ \pm}, C_{2}^{ \pm}, C_{3}^{ \pm}$, and $s_{1}$; for convenience we write $\xi= \pm$ instead of $\xi= \pm 1$ in sub- or superscripts. These are constrained by continuity and smoothness conditions at $y=0$ (the center of the source) and by matching with the leading order forms of the adjacent sinks. We first consider the conditions at $y=0$. Although the leading order (isolated) source solution (16) is continuous at $y=0$, it is not smooth. Therefore direct matching of the solutions for $\xi= \pm 1$ at $y=0$ is not possible. Rather, a transition layer is required, for which the appropriate rescalings are

$$
\widetilde{r}=\widehat{r} / \epsilon, \quad \widetilde{\psi}=\epsilon \widehat{\psi}, \quad \eta=y / \epsilon=z /(\epsilon \sqrt{2}) .
$$

Substituting these rescalings together with (6), (8), and (10) into (5) gives

$$
\begin{aligned}
d^{2} \widetilde{r} / d \eta^{2}-2 \widetilde{r} \widetilde{\psi}^{2} & =O\left(\epsilon^{2}\right), \\
d \widetilde{\psi} / d \eta+2(\widetilde{\psi} / \widetilde{r}) d \widetilde{r} / d \eta & =O\left(\epsilon^{2}\right) .
\end{aligned}
$$

Writing $\widetilde{r}=\widetilde{r}_{0}(y)+\epsilon \widetilde{r}_{1}(y)+\epsilon^{2} \widetilde{r}_{2}(y)+\cdots$ and $\widetilde{\psi}=\widetilde{\psi}_{0}(y)+\epsilon \widetilde{\psi}_{1}(y)+\epsilon^{2} \widetilde{\psi}_{2}(y)+\cdots$ and equating coefficients of $\epsilon^{0}$ and $\epsilon^{1}$ gives simple ODEs for the leading- and first order terms, with solutions

$$
\begin{aligned}
& \widetilde{r}_{0}=\left(A_{0}^{2}+2 B_{0}^{2} \eta^{2}\right)^{1 / 2}, \\
& \widetilde{\psi}_{0}=A_{0} B_{0} /\left(A_{0}^{2}+2 B_{0}^{2} \eta^{2}\right), \\
& \widetilde{r}_{1}=\left(A_{0} A_{1}+2 B_{0} B_{1} \eta^{2}\right) /\left(A_{0}^{2}+2 B_{0}^{2} \eta^{2}\right)^{1 / 2}, \\
& \widetilde{\psi}_{1}=\left[\left(A_{0}^{3} B_{1}-A_{0}^{2} B_{0} A_{1}\right)+2\left(B_{0}^{3} A_{1}-A_{0} B_{0}^{2} B_{1}\right) \eta^{2}\right] /\left[A_{0}^{2}+2 B_{0}^{2} \eta^{2}\right]^{2} .
\end{aligned}
$$

Copyright (C) by SIAM. Unauthorized reproduction of this article is prohibited. 
Here $A_{0}, B_{0}, A_{1}$, and $B_{1}$ are constants of integration; we take $B_{0}>0$ for uniqueness. The two additional constants of integration correspond to $O(\epsilon)$ and $O\left(\epsilon^{2}\right)$ translations in $\eta$, and these are determined by our requirement that $\widehat{r}$ have a local minimum at $z=0$, which implies that $d \widetilde{r}_{0} / d \eta=d \widetilde{r}_{1} / d \eta=0$ at $\eta=0$. Note that $\widetilde{\psi}_{0}$ has a local maximum at $\eta=0$, implying that $\psi$ has a spike of width $O(\epsilon)$ and height $B_{0} /\left(\epsilon A_{0}\right)$ at the centers of the sources. In section 5.1, we present numerical solutions that corroborate this feature of the solutions.

The behavior of (21) as $\eta \rightarrow \pm \infty$ is

$$
\begin{aligned}
\widetilde{r}_{0} & =\sqrt{2} B_{0}|\eta|+O(1 / \eta), \\
\widetilde{\psi}_{0} & =A_{0} /\left(2 B_{0} \eta^{2}\right)+O\left(1 / \eta^{4}\right), \\
\widetilde{r}_{1} & =\sqrt{2} B_{1}|\eta|+O(1 / \eta), \\
\widetilde{\psi}_{1} & =O\left(1 / \eta^{2}\right) .
\end{aligned}
$$

These behaviors must match with those of $\widehat{r}_{0}(y)+\epsilon \widehat{r}_{1}(y)$ and $\widehat{\psi}_{0}(y)+\epsilon \widehat{\psi}_{1}(y)$ as $y \rightarrow 0$. The expansions

$$
\widehat{r}_{0}=\xi R^{*} y+O\left(y^{3}\right) \text { and } \widehat{\psi}_{0}=-\sqrt{1-R^{* 2}} y+O\left(y^{3}\right)
$$

follow immediately from (16). Explicit differentiation of (19) implies that

$$
\widehat{r}_{1}(y)=C_{3}^{\xi}+\operatorname{Re}\left[F\left(\alpha, \beta, \gamma ; \frac{1}{2}\right)\right]\left(C_{1}^{\xi}+C_{2}^{\xi}-\xi H_{1}\right) y+O\left(y^{2}\right)
$$

as $\xi y \rightarrow 0^{+}$; a formula for the constant $H_{1}$ as a function of $R^{*}$ (or, equivalently, of $c$ ) is given in Appendix A. Initial calculation of the expansion (23) gives an additional term - $\operatorname{Re}\left[F\left(\alpha, \beta, \gamma ; \frac{1}{2}\right)\right] s_{1} H_{2}$, where $H_{2}$ is a combination of integrals involving the hypergeometric function, which is given explicitly in Appendix A. However, careful investigation (see Appendix A) shows that $\mathrm{H}_{2}$ is in fact identically zero.

For $\widehat{\psi}_{1}$, expanding the various terms in (17a) in power series and simplifying using (18) and (19) gives

$$
\widehat{\psi}_{1}=\frac{-\left[I_{1}+\xi s_{1} I_{2}+C_{1}^{\xi}-C_{2}^{\xi}\right] \operatorname{Re}\left[F^{\prime}\left(\alpha, \beta, \gamma ; \frac{1}{2}\right)\right]-2 s_{1} \xi R^{*} \sqrt{2}}{8 \xi R^{*} \sqrt{1-R^{* 2}}} y^{-2}-\frac{\xi C_{3}^{\xi} \sqrt{1-R^{* 2}}}{R^{*}}+O(y)
$$

as $\xi y \rightarrow 0^{+}$; formulae for the constants $I_{1}$ and $I_{2}$ as functions of $R^{*}$ (or, equivalently, of $c$ ) are given in Appendix A.

With these expansions, matching of the transition layer and "outer" solutions is straightforward. Using the intermediate variable $y_{\nu}=y / \nu(\epsilon)=\eta \epsilon / \nu(\epsilon)$ with $1 \gg \nu \gg \epsilon$, we require that as $\epsilon \rightarrow 0$ with $y_{\nu}$ fixed,

$$
\begin{aligned}
\widehat{r}_{0}\left(\nu y_{\nu}\right)+\epsilon \widehat{r}_{1}\left(\nu y_{\nu}\right) & =\epsilon \widetilde{r}_{0}\left(\nu y_{\nu} / \epsilon\right)+\epsilon^{2} \widetilde{r}_{1}\left(\nu y_{\nu} / \epsilon\right)+O\left(\epsilon^{3}\right), \\
\epsilon \widehat{\psi}_{0}\left(\nu y_{\nu}\right)+\epsilon^{2} \widehat{\psi}_{1}\left(\nu y_{\nu}\right) & =\widetilde{\psi}_{0}\left(\nu y_{\nu} / \epsilon\right)+\epsilon \widetilde{\psi}_{1}\left(\nu y_{\nu} / \epsilon\right)+O\left(\epsilon^{3}\right) .
\end{aligned}
$$

Copyright (c) by SIAM. Unauthorized reproduction of this article is prohibited. 
These give the conditions

$$
\begin{aligned}
& C_{3}^{\xi}=0 \\
& B_{0}=\frac{R^{*}}{\sqrt{2}} \\
& B_{1}=\operatorname{Re}\left[F\left(\alpha, \beta, \gamma ; \frac{1}{2}\right)\right] \frac{C_{1}^{\xi}+C_{2}^{\xi}-\xi H_{1}}{\xi \sqrt{2}} \\
& A_{0}=-2 B_{0}\left(\frac{\left[I_{1}+\xi s_{1} I_{2}+C_{1}^{\xi}-C_{2}^{\xi}\right] \operatorname{Re}\left[F^{\prime}\left(\alpha, \beta, \gamma ; \frac{1}{2}\right)\right]+2 s_{1} \xi R^{*} \sqrt{2}}{8 \xi R^{*} \sqrt{1-R^{* 2}}}\right) .
\end{aligned}
$$

Note that the leading order term in $\widehat{\psi}_{0}$ is not determined by matching at this order; rather it will match with an $O_{s}(\eta)$ term in $\widetilde{\psi}_{2}(\eta)$. Here we use the notation $O_{s}$ in the usual way: $\delta_{1}=O_{s}\left(\delta_{2}\right) \Leftrightarrow \delta_{1}=O\left(\delta_{2}\right)$ and $\delta_{1} \neq o\left(\delta_{2}\right)$. Conditions (26)-(28) determine $A_{0}, B_{0}$, and $B_{1}$ in terms of the seven undetermined constants in (19); $A_{1}$ is determined by higher order matching. However, more significantly, (25) determines $C_{3}^{+}$and $C_{3}^{-}$(both zero), while the right-hand sides of (27) and (28) must be invariant under $\xi \rightarrow-\xi$ since the left-hand sides are independent of $\xi$. Therefore,

$$
\begin{aligned}
-C_{1}^{-}-C_{2}^{-} & =C_{1}^{+}+C_{2}^{+}, \\
-I_{1}-C_{1}^{-}+C_{2}^{-} & =I_{1}+C_{1}^{+}-C_{2}^{+} .
\end{aligned}
$$

We now consider matching the perturbed source solution to the leading order sink solution, which will give four further conditions to be satisfied by the constants and by the source-sink separation $L_{\xi}(\epsilon)$. Standard asymptotic behavior of the hypergeometric function can be used to derive the leading order behavior of (19) as $y \rightarrow \xi \infty$; details of this are given in appendices of both [50] and [54]. This implies that

$$
\widehat{r}_{1}(y)=C_{1}^{\xi} \mathrm{e}^{\xi y} \operatorname{Re}\left[Q_{\xi} \mathrm{e}^{i \delta \xi y}\right]-C_{2}^{\xi} \mathrm{e}^{\xi y} \operatorname{Re}\left[Q_{-\xi} \mathrm{e}^{i \delta \xi y}\right]+O(1)
$$

as $y \rightarrow \xi \infty$; formulae for the constants $Q_{ \pm}$as functions of $R^{*}$ (or, equivalently, of $c$ ) are given in Appendix A.

3.2. Decay of the sink to the asymptotic wavetrain. In contrast to the source, we do not have an explicit form for the leading order (isolated) sink solution. Indeed to our knowledge, the existence of such a solution has not been proved, except when $c \ll 1$ (Doelman [14], Kapitula [21]). Counting stable and unstable manifold dimensions of the wavetrains as equilibria suggests that, generically, one should expect a two-parameter family of sink solutions of (1) (van Saarloos and Hohenberg [60]); typical parameters would be the amplitudes of the two asymptotic wavetrains. Numerical evidence suggests that this generic behavior does occur (e.g., Bohr, Huber, and Ott [3]). Therefore, we assume that there is exactly one (stationary) sink solution for which both asymptotic wavetrains have amplitude $R^{*}$; we have performed a detailed numerical confirmation of this (see Appendix B). The eigenvalue structure of (15)

Copyright (C) by SIAM. Unauthorized reproduction of this article is prohibited. 
at $\widehat{r}_{0}=R^{*}, \widehat{\psi}_{0}=-\xi \sqrt{1-R^{* 2}}$ implies that the amplitude of this sink solution, $r_{\text {sink }}(y)$ say, must satisfy

$$
r_{\text {sink }}(y)-R^{*}=\operatorname{Re}\left[S \exp \left(-(1 / \sqrt{2}+i \delta / \sqrt{2}) z_{\text {sink }}\right)\right]+o\left(e^{-z_{\text {sink }} / \sqrt{2}}\right)
$$

as $z_{\text {sink }} \rightarrow \infty$. Here the coordinate $z_{\text {sink }}>0$ is measured from the center of the sink, so that $z_{\operatorname{sink}}=L_{\xi}(\epsilon)-\xi y \sqrt{2} . S$ is a complex valued constant that is known in principle, but an explicit form for its dependence on $R^{*}$ (or equivalently on $c$ ) is not available without an exact isolated sink solution. However, $S$ can be estimated very accurately via numerical solutions of (5); details of this are given in Appendix B. Note that because the isolated sink solution is necessarily symmetric in $r$ (and antisymmetric in $\psi$ ), $S$ is the same for $\xi=-1$ and $\xi=+1$.

3.3. Matching source and sinks. Matching requires that

$$
\widehat{r}_{0}(y)+\epsilon \widehat{r}_{1}(y)=r_{\operatorname{sink}}(y)+o(\epsilon)
$$

when $1 \ll y \ll L_{\xi}(\epsilon) / \sqrt{2}$ as $\epsilon \rightarrow 0$, i.e.,

$$
\begin{aligned}
R^{*} & +\epsilon C_{1}^{\xi} \mathrm{e}^{\xi y} \operatorname{Re}\left[Q_{\xi} \mathrm{e}^{i \delta \xi y}\right]-\epsilon C_{2}^{\xi} \mathrm{e}^{\xi y} \operatorname{Re}\left[Q_{-\xi} \mathrm{e}^{i \delta \xi y}\right]+o\left(\epsilon \mathrm{e}^{\xi y}\right) \\
& =R^{*}+\mathrm{e}^{\xi y} \operatorname{Re}\left[S \exp \left(i \xi \delta y-(1+i \delta) L_{\xi}(\epsilon) / \sqrt{2}\right)\right]+o\left(\mathrm{e}^{\xi y-L_{\xi}(\epsilon) / \sqrt{2}}\right)+o(\epsilon) .
\end{aligned}
$$

Since this equation must hold for a range of $y$ values, it requires that

$$
\epsilon C_{1}^{\xi} Q_{\xi}-\epsilon C_{2}^{\xi} Q_{-\xi}=S \exp \left(-(1+i \delta) L_{\xi}(\epsilon) / \sqrt{2}\right)+o\left(\mathrm{e}^{-L_{\xi}(\epsilon) / \sqrt{2}}\right)+o(\epsilon) .
$$

Equation (32) is complex valued and must hold for $\xi=+1$ and $\xi=-1$; therefore it constitutes four additional conditions.

There are two distinct classes of solution to (32). One possibility is

$$
\begin{aligned}
\mathrm{e}^{-L_{\xi}(\epsilon) / \sqrt{2}} & =o(\epsilon) \\
\text { and } \quad C_{1}^{\xi}=C_{2}^{\xi} & =0 ;
\end{aligned}
$$

the second of these follows from the first because $Q_{ \pm}$are linearly independent complex numbers for all $R^{*} \in(0,1)$. The alternative possibility is

$$
\begin{aligned}
\mathrm{e}^{-L_{\xi}(\epsilon) / \sqrt{2}} & =\kappa_{\xi}|\epsilon| \\
\text { and } \quad C_{1}^{\xi} Q_{\xi}-C_{2}^{\xi} Q_{-\xi} & =\kappa_{\xi} \operatorname{sign}(\epsilon) S \exp \left\{i \delta\left(\log |\epsilon|+\log \kappa_{\xi}\right)\right\}+o(1)
\end{aligned}
$$

as $\epsilon \rightarrow 0$ for some $\kappa_{\xi}>0$ that is $O_{s}(1)$. Now fix a sign $\zeta \in\{ \pm 1\}$ (using $\zeta= \pm,-\zeta=\mp$ in sub- and superscripts). If (33) holds for $\xi=\zeta$, then (29) implies that

$$
\begin{aligned}
& C_{1}^{-\zeta}+C_{2}^{-\zeta}=0, \\
& C_{1}^{-\zeta}-C_{2}^{-\zeta}=-2 I_{1} .
\end{aligned}
$$

Copyright (c) by SIAM. Unauthorized reproduction of this article is prohibited. 
The formula for $I_{1}$ given in Appendix A implies that it is nonzero for all $R^{*} \in(0,1)$, so that (35) is incompatible with (33b) for $\xi=-\zeta$ for any value of $s_{1}$. Hence, (33) cannot hold for both $\xi=\zeta$ and $\xi=-\zeta$. Thus if (33) holds for $\xi=\zeta$, condition (34b) would then have to hold for $\xi=-\zeta$, with $C_{1}^{-\zeta}$ and $C_{2}^{-\zeta}$ determined by (35), which requires

$$
\begin{aligned}
\kappa_{-\zeta} \exp \left(+i \delta \log \kappa_{-\zeta}\right)=\Upsilon & \equiv \widetilde{\Upsilon} \operatorname{sign}(\epsilon) \exp (-i \delta \log |\epsilon|), \\
\text { where } \widetilde{\Upsilon} & =-I_{1}\left(Q_{+}+Q_{-}\right) / S .
\end{aligned}
$$

This holds for some $\kappa_{-\zeta}>0$ if and only if

$$
(\delta \log |\Upsilon|-\arg \Upsilon) / 2 \pi \in \mathbb{Z}
$$

In conclusion, except in the special case of (37) being satisfied, condition (34) must hold for $\xi=+1$ and $\xi=-1$, implying that to leading order the source-sink separation is given by $-\sqrt{2} \log |\epsilon|$. We can then solve (34b) for the leading order forms of $C_{1}^{\xi}$ and $C_{2}^{\xi}$; these are given in Appendix A. Substituting these back into (29), we obtain two conditions on $\kappa_{+}$and $\kappa_{-}$. These can be expressed as linear equations for the real and imaginary parts of $\left(\kappa_{+}^{1-i \delta}-\kappa_{-}^{1-i \delta}\right)$, whose solution yields the single complex condition

$$
\kappa_{-} \exp \left(+i \delta \log \kappa_{-}\right)-\Upsilon / 2=-\kappa_{+} \exp \left(+i \delta \log \kappa_{+}\right)+\Upsilon / 2
$$

to leading order, with a correction that is $o(1)$ as $\epsilon \rightarrow 0 ; \Upsilon$ is defined in (36). The left(right-) hand sides of (38) trace out logarithmic spirals in the complex plane as $\kappa_{-}\left(\kappa_{+}\right)$varies between 0 and $+\infty$. We will refer to these spirals as $\mathcal{S}_{ \pm}$; they are point reflections of one another through the origin, with centers at $\pm \Upsilon / 2$ and with the same orientation. Solutions for $\kappa_{-}$and $\kappa_{+}$occur when $\mathcal{S}_{ \pm}$intersect.

Condition (34) requires that $\kappa_{ \pm}>0$; however, we allow $\kappa_{ \pm}=0$ in (38); this corresponds to (37), and thus (38) incorporates both matching conditions, with $L_{ \pm} \sim-\sqrt{2} \log |\epsilon|$ as $\epsilon \rightarrow 0$ if $\kappa_{ \pm} \neq 0$, and $L_{ \pm} \gg-\sqrt{2} \log |\epsilon|$ if $\kappa_{ \pm}=0$. In the latter case there is an $O(\epsilon)$ contribution to the solution for $\mp y>0$, but for $\pm y>0$ the correction to the leading order (isolated) source solution is $o(\epsilon)$. Investigation of the source-sink separation in this case would require higher order matching, which we have not attempted.

If (37) holds, then $\mathcal{S}_{+}$has its center point on $\mathcal{S}_{-}$(and vice versa), so that the two spirals intersect an infinite number of times. In Appendix D we show that, otherwise, the number of intersections is finite and can be zero. A simple change of variables (given in (D.1)) shows that the number of intersections depends on $\Upsilon$ via the real quantity

$$
\frac{\delta \log |\Upsilon|-\arg \Upsilon}{2 \pi}=\frac{\delta \log |\epsilon|}{2 \pi}+\frac{1}{4}(\operatorname{sign}(\epsilon)-1)+\frac{\delta \log |\widetilde{\Upsilon}|-\arg \widetilde{\Upsilon}}{2 \pi} ;
$$

recall that $\widetilde{\Upsilon}$, which is defined in (36), is independent of $\epsilon$. Moreover, the number of intersections has a self-similarity: if $\left(\kappa_{-}^{*}, \kappa_{+}^{*}\right)$ is a solution for $\Upsilon=\Upsilon^{*}$, then $\left(\kappa_{-}^{*} \mathrm{e}^{2 n \pi / \delta}, \kappa_{+}^{*} \mathrm{e}^{2 n \pi / \delta}\right)$ is a solution for $\Upsilon=\Upsilon^{*} \mathrm{e}^{2 n \pi / \delta}$ for any integer $n$. Therefore, for a given value of $R^{*}$ (or, equivalently, of $c$ ) and for either value of $\operatorname{sign}(\epsilon)$, the set $\mathcal{S}_{+} \cap \mathcal{S}_{-}$is periodic in $\log |\epsilon|$, with period $2 \pi / \delta$. In addition, it is the same for $\epsilon=\epsilon^{*}>0$ and $\epsilon=-\epsilon^{*} \mathrm{e}^{\pi / \delta}$. As discussed in section 2, the

Copyright (c) by SIAM. Unauthorized reproduction of this article is prohibited. 
(a)

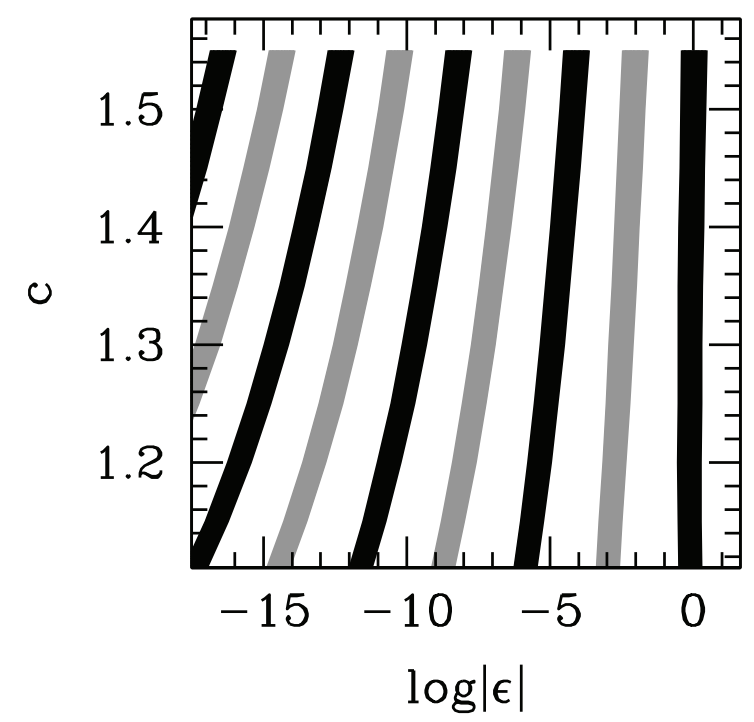

(b)

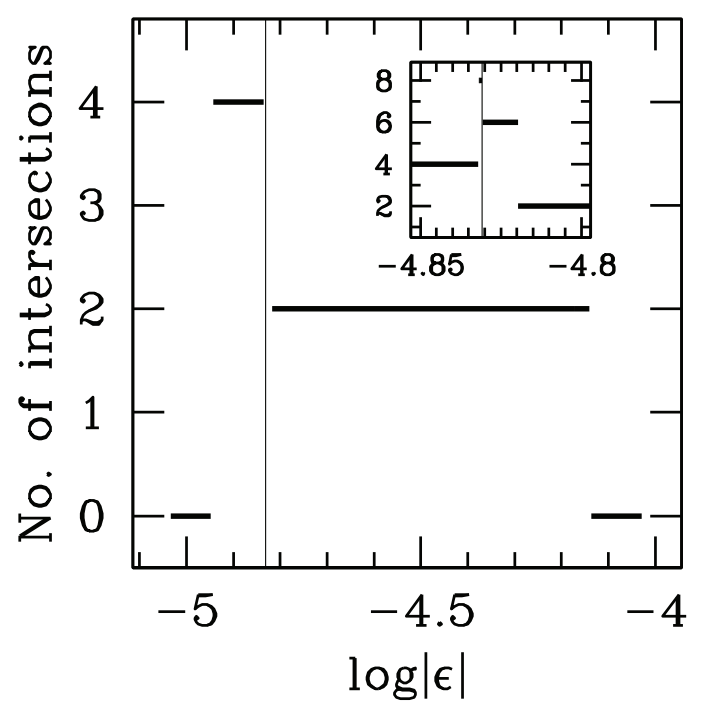

Figure 5. An illustration of the number of possible intersections of the spirals $\mathcal{S}_{ \pm 1}$, as a function of $\epsilon$ and c. Each intersection corresponds to a family of patterns in which the speed coefficient $s_{1}$ is an unconstrained parameter. (a) The values of $\epsilon$ for which there is at least one pair of intersections. We plot $\log |\epsilon|$ on the horizontal axis, and indicate regions (shaded) in which there are patterns. Dark/light shading indicates a pattern with $\epsilon$ positive/negative. (b) A more detailed plot on a small region of the $\log |\epsilon|$ axis, for $c=1.4$, showing the number of intersections. These are all for $\epsilon>0$. The number of intersections has a sharp peak centered around the value of $\epsilon$ for which (37) is satisfied (indicated by a thin vertical line). There it is infinite, and the center of each spiral lies on the other. By symmetry, the number of intersections is even except at values of $\epsilon$ for which there is a root of (38) with $\kappa_{+}=\kappa_{-}$. This occurs once in each shaded band in (a), when $[\delta \log (|\Upsilon| / 2)-\arg \Upsilon] / 2 \pi \in \mathbb{Z}$; in (b), there is exactly one intersection when $\log |\epsilon|=-4.1377$. Numerical values of $\Upsilon$ are required for these plots. Formulae for $H_{1}, I_{1}$, and $Q_{ \pm}$are given in Appendix A; MAPLE code that evaluates these formulae numerically is given in 78096_01.zip [82.2KB]. Numerical calculation of $S$ is described in Appendix B. In (a), the range of $c$ on the vertical axis corresponds to the wavetrain of amplitude $R^{*}$ being unstable but absolutely stable.

wavetrain of amplitude $R^{*}$ is unstable but absolutely stable when $1.110468<c<1.576465$. For $c$ in this range, the number of intersections of the two spirals is zero for a sequence of nontrivial intervals of $\epsilon$ values, separated by intervals in which there are one or more pairs of intersections (illustrated in Figure 5). This implies that, although some values of $\epsilon$ allow patterns with multiple source-sink separations (even an infinite number, if (37) is satisfied), for many other values of $\epsilon$ there are no possible patterns.

4. Families of source-sink patterns. Condition (38) for $\kappa_{ \pm}$completes the leading order matching. Note that at this order, the regions between two neighboring sinks are completely decoupled from one another, although we expect there to be coupling at lower order. Our calculations suggest that there is a three-parameter family of patterns of sources and sinks. One parameter is $\epsilon$; recall that this corresponds to the difference between $R^{*}$ and the average of the asymptotic wavetrain amplitudes on the two sides of a source or sink. The value of $\epsilon$ is constrained to be such that (38) has at least one pair of solutions for $\kappa_{ \pm}$, which occurs 
in nontrivial intervals (see Figure 5). A second parameter is the constant of proportionality $s_{1}$ between the speed of movement and $\epsilon$. This is totally absent in the majority of the above calculation and has essentially no effect on the solution structure -in particular, any dependence of the source-sink separations on $s_{1}$ is $o(1)$ as $\epsilon \rightarrow 0$. Recall from (12) that specifying $\epsilon$ and $s_{1}$ is equivalent to specifying the asymptotic wavetrain amplitudes on the two sides of the source. These first two parameters of the family are continuous, but the third is discrete and comes from the possible multiplicity of solutions for $\kappa_{ \pm}$that is implied by (38). Recall that $\kappa_{-}$and $\kappa_{+}$determine the $O_{s}(1)$ part of the separation between a source and a neighboring sink on its right and left, respectively, with the separations being

$$
L_{ \pm}=-\sqrt{2} \log |\epsilon|-\sqrt{2} \log \kappa_{ \pm}+o(1)
$$

as $\epsilon \rightarrow 0$.

Interpretation of this solution family is somewhat easier if one reformulates the problem as follows: given distances $L_{ \pm}$, is there a source-sink pattern with separations $L_{ \pm}$between a source and the neighboring sinks? Our calculation answers this question for $L_{ \pm} \rightarrow \infty$ with $L_{+}-L_{-}=O_{s}(1) \cdot{ }^{1}$ Multiplying (38) by $|\epsilon|$ and using (34a) gives

$$
\exp \left(-L_{-}(1+i \delta) / \sqrt{2}\right)+\exp \left(-L_{+}(1+i \delta) / \sqrt{2}\right)=\epsilon \widetilde{\Upsilon}+o(\epsilon)
$$

as $\epsilon \rightarrow 0$, where $\widetilde{\Upsilon}$ is defined in (36). This constitutes two (real) equations in the three unknowns $L_{ \pm}$and $\epsilon$, and we expect curves of solutions in $\left(L_{-}, L_{+}, \epsilon\right)$-space. Indeed, a sinksource-sink pattern is a heteroclinic connection of the same type as a sink, which is (generically) robust. Note that $\widetilde{\Upsilon}$ is independent of $\epsilon$; it is a function of $R^{*}$ (or, equivalently, of $c$ ). Thus geometrically, in the complex plane, the right-hand side forms a line through the origin parameterized by $\epsilon$, and for fixed $L_{-}$, say, the left-hand side is a logarithmic spiral parameterized by $L_{+}$. The formula is valid near the origin, corresponding to $L_{+}$and $L_{-}$being sufficiently large. Analytically, a pattern exists if $L_{ \pm}$satisfy

$$
\arg \left[\exp \left(-L_{-}(1+i \delta) / \sqrt{2}\right)+\exp \left(-L_{+}(1+i \delta) / \sqrt{2}\right)\right]=\arg [\operatorname{sign}(\epsilon) \tilde{\Upsilon}]+o(1)
$$

as $\epsilon \rightarrow 0$ or, equivalently, as $L_{ \pm} \rightarrow \infty$ with $L_{+}-L_{-}=O_{s}(1)$. Equation (40) is a "locking condition," which ensures appropriate phase matching of the oscillatory decay of the sinks to their asymptotic wavetrains. Similar conditions apply for interacting pulses in reactiondiffusion equations (see, for example, Sandstede and Scheel [47]). That situation is analogous to the case $L_{+}=\infty$ or $L_{-}=\infty$, and the locking condition arises as a condition for stability only. In contrast, in our case (40) must hold for the solution to even exist. Given any solution pair $L_{ \pm}^{*}$ of $(40), L_{ \pm}^{*}+2 \sqrt{2} n \pi / \delta$ is also a solution for any $n \in \mathbb{Z}$, with the same sign for $\epsilon$. Therefore (40) defines a sequence of curves in the $\left(L_{-}, L_{+}\right)$-plane (Figure 6(a)). Each curve approaches a limiting value of $L_{ \pm}$as $L_{\mp 1} \rightarrow \infty$. In this limit, (37) is satisfied, and (40) implies that the limiting source-sink separations $L$ are

$$
L=(\sqrt{2} / \delta) \cdot[n \pi-\arg (\widetilde{\Upsilon})] \quad(n \in \mathbb{Z}, n>\arg (\widetilde{\Upsilon}) / \pi) .
$$

\footnotetext{
${ }^{1}$ Our calculation also applies for $L_{ \pm} \rightarrow \infty$ with $L_{\mp 1}=\infty$, which corresponds to (37) being satisfied.
} 

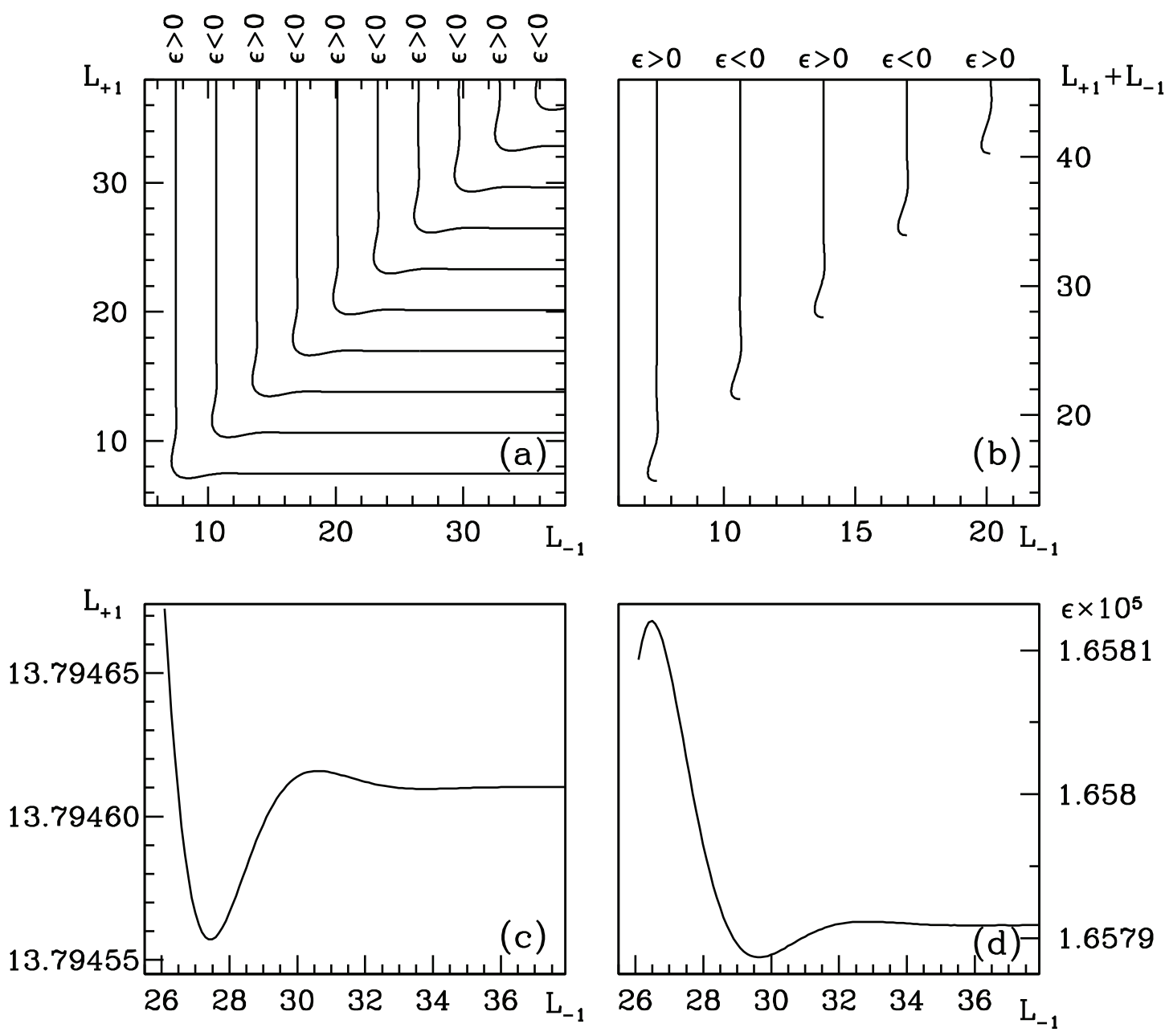

Figure 6. An illustration of the phase locking condition (40) that must be satisfied by $L_{ \pm}$for a sink-sourcesink pattern to exist, for $c=1.4$. The condition implies a sequence of curves in the $L_{+}-L_{-}$plane, which are shown in (a). The corresponding values of $\epsilon$ have a fixed sign along each curve, which alternates through the sequence as indicated. (b) shows a different visualization, plotting one of the separation distances against their sum, which is the separation of adjacent sinks. The approach of each solution curve to its limiting value as $L_{+}$ or $L_{-} \rightarrow \infty$ is oscillatory, and we illustrate this in (c). Finally, in (d) we plot the variation of $\epsilon$ along one of the curves (the same one as used in (c)); the value of $\epsilon$ is given as a function of $L_{ \pm}$by (42), with $\operatorname{sign}(\epsilon)$ chosen as in (40).

The approach to these limiting values is oscillatory (Figure 6(b)), corresponding to the spirals $\mathcal{S}_{ \pm 1}$ winding in towards their centers. The limiting values (41) are the separations that are possible in a solution consisting a single source-sink pair; their variation with $c$ is illustrated in Figure 7.

If (40) is satisfied, then $\epsilon$ is uniquely determined via

$$
|\epsilon|=\left|\exp \left(-L_{-}(1+i \delta) / \sqrt{2}\right)+\exp \left(-L_{+}(1+i \delta) / \sqrt{2}\right)\right| /|\widetilde{\Upsilon}|
$$

Copyright (c) by SIAM. Unauthorized reproduction of this article is prohibited. 


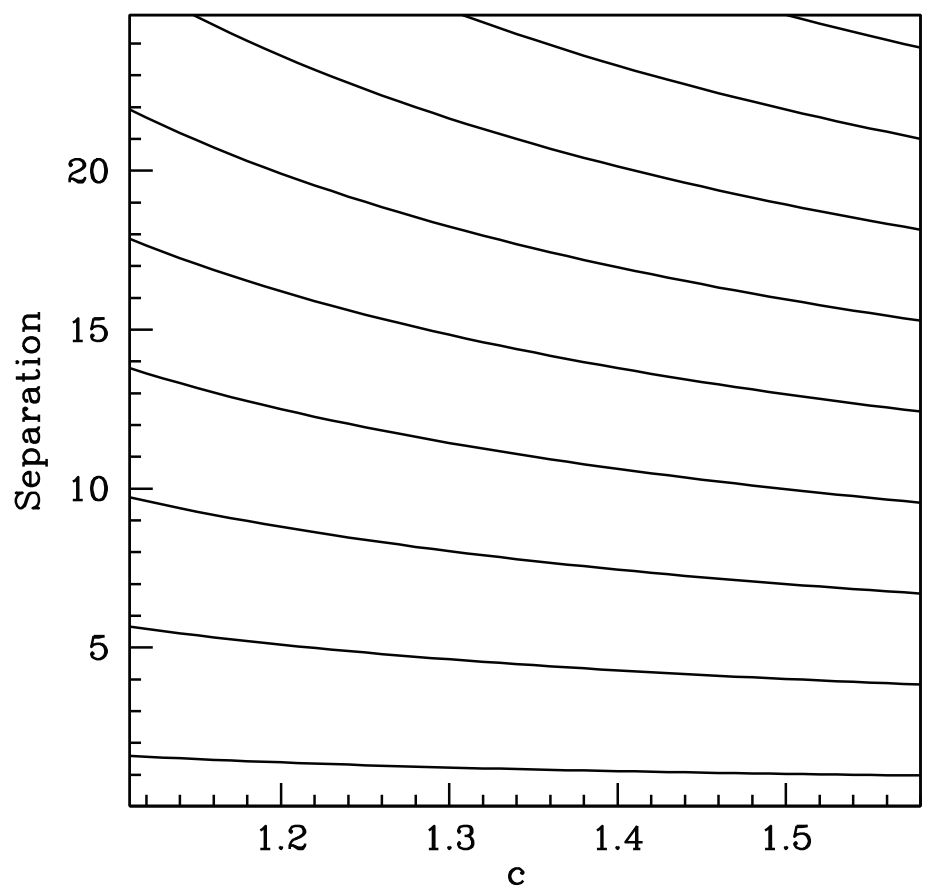

Figure 7. An illustration of the dependence on $c$ of the possible separations between the source and sink in an isolated source-sink pair. This corresponds to the phase locking condition (40) being satisfied with one of $L_{ \pm}$being infinite. These separations are given by the formula (41).

with $\operatorname{sign}(\epsilon)$ being the same as in (40). Each of the solution curves shown in Figure 6(a) traces out one of the $\epsilon$ intervals shown in Figure 5(a), in which the spirals $\mathcal{S}_{+1}$ and $\mathcal{S}_{-1}$ intersect. In Figure 6(c) we show the variation of $\epsilon$ along one of the solution curves. The limiting value of $\epsilon$, corresponding to the separation (41), is

$$
\epsilon=(-1)^{n} \mathrm{e}^{-L / \sqrt{2}} /|\widetilde{\Upsilon}|
$$

The value of $\epsilon$ determines the average of the amplitudes of the asymptotic wavetrains on either side of the source. The difference between these amplitudes, or equivalently the propagation speed of the source-sink pattern, is arbitrary, except that for our calculation to be valid it must be $O\left(\mathrm{e}^{-L_{ \pm}}\right)$as $L_{ \pm} \rightarrow \infty$.

These considerations specify the possible solutions between two successive sinks (with a source in between them). Finally, we comment that there can be multiple distinct pairs $L_{ \pm}$ satisfying (40) and giving the same value of $\epsilon$ (see Figures 5(b) and 6(d)). Since at first order each sink-sink block is decoupled, it is possible that they can be glued together to give a rich variety of composite patterns. Investigation of this would require higher order matching, which we have not attempted.

\section{Numerical verification.}

5.1. ODE simulations. In section 5.2, we will present results from numerical solutions of the PDEs (1) demonstrating the discreteness of the possible separation distances in source-sink 
patterns. However, the numerical accuracy of our PDE simulations is not sufficient to enable a detailed quantitative test of the analytical results derived in section 2. For that, we used instead numerical solutions of the travelling wave ODEs (5), with $d \widehat{\theta}(t) / d t=K_{0}+\epsilon$ and $c=\epsilon s_{1}$; $K_{0}$ is given in (10). Using these equations, we calculate numerically, using 16-byte precision, solutions consisting of one source and one sink in an unbounded domain, which corresponds to $L_{+}$(say) being infinite; a typical example is shown in Figure 8. As $|z| \rightarrow \infty$, a solution of this type decays to the asymptotic wavetrain with $\widehat{\psi}<0$. Recall from section 3 that linearizing (5) about this wavetrain shows that it has one real eigenvalue and a complex conjugate pair; these and the corresponding eigenvectors can easily be calculated numerically. The approach to the asymptotic wavetrain is oscillatory as $z \rightarrow-\infty$ and monotonic as $z \rightarrow+\infty$. Therefore to construct numerical solutions, we solve (5) in the negative $z$ direction, starting with $\widehat{r}, d \widehat{r} / d z$, and $\widehat{\psi}$ on the eigenvector corresponding to the real (negative) eigenvalue. This reduces the problem to one of shooting in the single unknown parameter $\epsilon$, which must be chosen so that the solution traces out a source followed by a sink, and then decays again to the asymptotic wavetrain. Although it is simple in concept, this procedure is complicated in practice by the extreme sensitivity of the solutions of (5) to changes in $\epsilon$. For example, the solution shown in Figure 8 has $\epsilon \approx-2 \times 10^{-8}$ (see the legend for the precise value) and uses a domain of length 53. If $\epsilon$ is increased by more than $6 \times 10^{-11}$, or decreased by more than $10^{-14}$, the solution for $\widehat{r}$ increases through 1 before reaching the end of the domain, after which it rapidly increases towards infinity. For this reason, it is not feasible to attempt a purely numerical study of source-sink separations: the analytical results are an essential prerequisite to provide the appropriate values of $\epsilon$.

Table 1 compares six successive stationary source-sink pair solutions, calculated numerically in this way, against the analytical approximations derived in section 2 . The comparison is extremely good. There is numerical error in both the simulations and the predictions; the latter is due to error in the numerical calculation of the constant $S$. In Appendix C we present a detailed discussion of these errors and conclude that we have a high level of confidence in the first 10 decimal places of the separations and the first 10 significant figures of the values of $\epsilon$, in both the simulations and the predictions. For each of the cases presented in Table 1, the difference between the simulated and predicted separations is much greater than $10^{-10}$ and is therefore an accurate measure of the higher order corrections to our perturbation theory expansions. These corrections decrease with $\epsilon$, as predicted by the theory. Moreover, the entries in column 3 differ between parts (a) and (b) of Table 1, suggesting that the corrections do depend on $s_{1}$. This would imply that the speed of propagation of the sources and sinks does affect the possible separations, but with this dependence being $o(1)$ as $\epsilon \rightarrow 0$.

The ODE solutions contain a sharp spike in $\psi$ centered at the source (see Figure 8 ), as predicted by the theory. Our analysis predicts that this spike has height $B_{0} /\left(\epsilon A_{0}\right)+O(1)$ as $\epsilon \rightarrow 0$, with $B_{0}$ and $A_{0}$ given in (26) and (28), respectively. For a single source-sink pair,

$$
B_{0} /\left(\epsilon A_{0}\right)=-4 R^{*} \sqrt{1-R^{* 2}} /\left(\epsilon\left(I_{1}+s_{1} I_{2}\right) \operatorname{Re}\left[F^{\prime}\left(\alpha, \beta, \gamma ; \frac{1}{2}\right)\right]+2 \sqrt{2} s_{1} R^{*}\right) .
$$

In column 5 of Table 1 we give the heights implied by (44) and compare them with the heights of the spikes in our numerical solutions. The comparison is extremely good, and the difference between the predictions and simulations is roughly constant as $\epsilon$ varies (see column 6

Copyright (c) by SIAM. Unauthorized reproduction of this article is prohibited. 


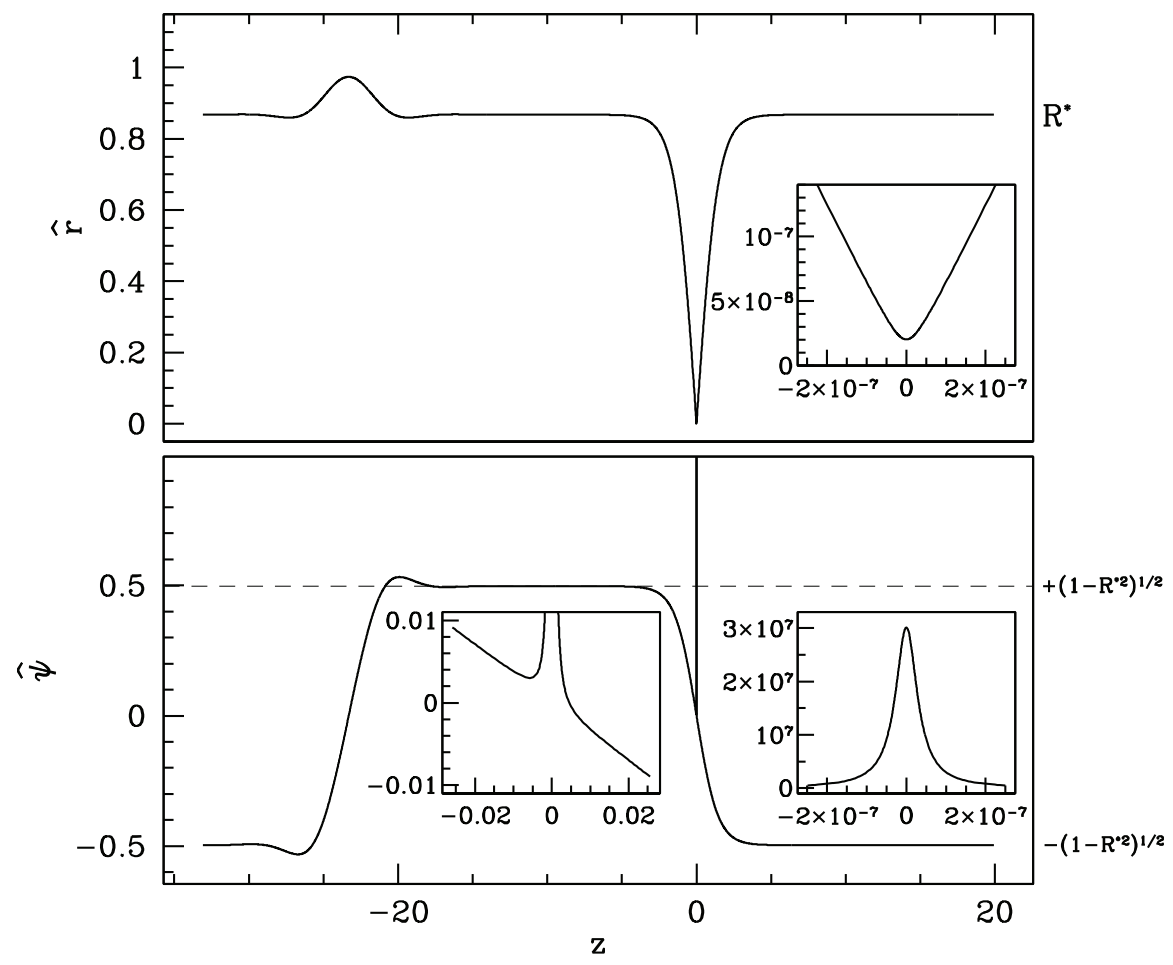

Figure 8. A numerical simulation of an isolated stationary source-sink pair, calculated using 16-byte precision. The insets show details of the solution near the center of the source. For $\psi$, we show this over two different ranges of $z$ : the left-hand inset shows the behavior near the base of the spike, while the righthand inset shows the spike in full. Note the very narrow ranges on the z-axis in the latter case and in the inset on the $r$ graph. The solution was obtained using (5) with $d \widehat{\theta}(t) / d t=K_{0}+\epsilon ; K_{0}$ is given in $(10)$. We integrated these equations backwards in z using the routine DLSODAR (Hindmarsh [18], Petzold [38]), which is part of the ODEPACK collection, and is freely available at http://www.netlib.org. The solver automatically switches between an Adams predictor-corrector method and a Gear backward differentiation formula method. We set both the absolute and relative error tolerances in the routine to $\ell=10^{-24}$. We used initial conditions lying on the (unique) real eigenvector of the asymptotic wavetrain, normalized so that $r$ was $\sqrt{\ell}$ below its asymptotic wavetrain value. (Note that the amplitude of the asymptotic wavetrain is different from $R^{*}$, since $\epsilon \neq 0$.) We discuss the reason for this choice of normalization, and the size of the resulting numerical errors, in Appendix B. Prior to plotting, we made a translation in $z$ so that the source was centered at $z=0$, since this is the formulation that we have adopted in our perturbation theory calculation. The parameters are $\epsilon=-1.98921217 \times 10^{-8}, c=1.4$, and $s_{1}=0$; in the main text we comment on the extreme sensitivity of the numerical solutions to $\epsilon$. We determined the value of $\epsilon$ by numerical shooting. The appropriate shooting condition is that $(\widehat{r}, d \widehat{r} / d z, \widehat{\psi})-\left(R^{*}, 0,-\left(1-R^{* 2}\right)^{1 / 2}\right)$ lies in the plane spanned by the real and imaginary parts of the eigenvector corresponding to the complex conjugate pair of eigenvalues at the asymptotic wavetrain. In practice it is convenient to perform a primitive continuation in $\epsilon$, gradually increasing the domain length from about 42 to 45 using the alternative shooting condition $\widehat{r}=R^{*}$, before finally applying the correct condition. The routine DLSODAR automatically stops integration when specified algebraic conditions are satisfied, and this enables precise identification of the centers of the source and sink.

of Table 1), as predicted by the theory. It is important to emphasize that although the sourcesink separations have only a $o(1)$ dependence on $\epsilon$ as this tends to zero, other aspects of the patterns vary with $\epsilon$ to leading order. This is highlighted by comparing column 5 of parts (a)

Copyright ( by SIAM. Unauthorized reproduction of this article is prohibited. 
Table 1

A comparison of the predictions of our perturbation theory calculation and numerical simulations, performed using 16-byte precision, for a single stationary source-sink pair. Column 1 shows six successive predicted separations, given by solving (40) with one of $L_{+}$and $L_{-}$set to infinity, and column 2 shows the corresponding values of $\epsilon$, given by (42), with the sign of $\epsilon$ as in (40). Columns 3 and 4 show the differences between these predictions and the results from numerical solutions of (5), performed as discussed in the main text and in the legend to Figure 8. Accurate computation of the source-sink separations in these solutions is straightforward because of the root-finding capability of our ODE solver (see the legend to Figure 8). Column 5 shows the predicted height of the spike in $\psi$, given by (44), and column 6 shows the difference between this and the results from numerical simulations. The parameters are $c=1.4$ and (a) $s_{1}=0 ;$ (b) $s_{1}=1$. Only the first few significant figures are shown in each column, but we have a high level of confidence in the first 10 decimal places of the separations, and the first 10 significant figures of the values of $\epsilon$, for both the predictions and the simulations (see Appendix C). For the height of the spike in $\psi$, the estimated numerical error in simulations is at most $10^{-5}$ (see Appendix C), while the predicted values are free of numerical error, being independent of $S$.

\begin{tabular}{|c|c|c|c|c|c|}
\hline $\begin{array}{l}\text { Separation } \\
\left(\text { pred }^{\mathrm{n}}\right)\end{array}$ & $\begin{array}{c}\epsilon \\
\text { (prediction) }\end{array}$ & $\begin{array}{c}\text { Prediction } \\
-\operatorname{sim}^{\mathrm{n}} \\
\text { (separation) }\end{array}$ & $\begin{array}{c}\text { Prediction } \\
-\operatorname{sim}^{\mathrm{n}} \\
(\epsilon) \\
\end{array}$ & $\begin{array}{l}\text { Height of } \\
\text { spike in } \\
\psi\left(\text { pred }^{\mathrm{n}}\right) \\
\end{array}$ & $\begin{array}{c}\text { Pred }^{\mathrm{n}} \\
-\operatorname{sim}^{\mathrm{n}} \\
\text { (height) }\end{array}$ \\
\hline \multicolumn{6}{|c|}{ (a) $c=1.4, s_{1}=0$} \\
\hline 10.624 & $-1.560 \times 10^{-4}$ & $2.449 \times 10^{-3}$ & $5.517 \times 10^{-7}$ & $3.862 \times 10^{3}$ & 0.498 \\
\hline 13.795 & $1.658 \times 10^{-5}$ & $-3.222 \times 10^{-4}$ & $7.396 \times 10^{-9}$ & $-3.620 \times 10^{4}$ & 0.498 \\
\hline 16.965 & $-1.762 \times 10^{-6}$ & $4.091 \times 10^{-5}$ & $9.626 \times 10^{-11}$ & $3.408 \times 10^{5}$ & 0.498 \\
\hline 20.136 & $1.872 \times 10^{-7}$ & $-5.056 \times 10^{-6}$ & $1.232 \times 10^{-12}$ & $-3.207 \times 10^{6}$ & 0.500 \\
\hline 23.306 & $-1.989 \times 10^{-8}$ & $6.120 \times 10^{-7}$ & $1.555 \times 10^{-14}$ & $3.018 \times 10^{7}$ & 0.479 \\
\hline 26.476 & $2.114 \times 10^{-9}$ & $-7.352 \times 10^{-8}$ & $1.925 \times 10^{-16}$ & $-2.840 \times 10^{8}$ & 0.677 \\
\hline \multicolumn{6}{|c|}{ (b) $c=1.4, s_{1}=1$} \\
\hline 10.624 & $-1.560 \times 10^{-4}$ & $1.990 \times 10^{-3}$ & $2.152 \times 10^{-7}$ & $2.456 \times 10^{3}$ & 0.574 \\
\hline 13.795 & $1.658 \times 10^{-5}$ & $-2.536 \times 10^{-4}$ & $2.468 \times 10^{-9}$ & $-2.307 \times 10^{4}$ & 0.574 \\
\hline 16.965 & $-1.762 \times 10^{-6}$ & $3.159 \times 10^{-5}$ & $2.831 \times 10^{-11}$ & $2.171 \times 10^{5}$ & 0.574 \\
\hline 20.136 & $1.872 \times 10^{-7}$ & $-3.849 \times 10^{-6}$ & $3.244 \times 10^{-13}$ & $-2.043 \times 10^{6}$ & 0.576 \\
\hline 23.306 & $-1.989 \times 10^{-8}$ & $4.608 \times 10^{-7}$ & $3.733 \times 10^{-15}$ & $1.923 \times 10^{7}$ & 0.554 \\
\hline 26.476 & $2.114 \times 10^{-9}$ & $-5.501 \times 10^{-8}$ & $4.118 \times 10^{-17}$ & $-1.810 \times 10^{8}$ & 0.761 \\
\hline
\end{tabular}

and (b) of Table 1. For each of the possible separations, the heights of the spikes in $\psi$ differ by a factor of about 1.6 .

We conclude this subsection by commenting on the potential use of standard numerical continuation software to compute source-sink patterns. In principle this is a natural approach: for instance, one could track the patterns and the associated separation distances as $c$ varies (see Figure 7). However, we had great difficulties when attempting to perform such continuations for (5) using the software package AUTO (Doedel [12, 11], Doedel et al. [13]). One problem is the extreme sensitivity of the solutions of (5) to the parameter $\epsilon$, which necessitates extremely small continuation steps. But the second and more significant difficulty is the extremely sharp spike in $\widehat{\psi}$ : for example, in Figure $8, \widehat{\psi}$ increases by seven orders of magnitude over a $z$-interval of width about $10^{-7}$. Such spikes are a fundamental part of source-sink pattern solutions and pose enormous difficulties for continuation software: for instance, in AUTO, a very large number of collocation points would be required for accurate solutions.

5.2. PDE simulations. The basic prediction of our analytical theory is the discreteness of the possible separation distances in source-sink patterns. In section 1 we reported numerical

Copyright (c) by SIAM. Unauthorized reproduction of this article is prohibited. 
evidence of this phenomenon in simulations of the PDEs (1), which motivated our analytical work. In this subsection we describe more detailed and systematic numerical tests. The numerical solutions of the travelling wave ODEs (5) described in the previous subsection have a very high level of accuracy. However, our PDE simulations are generally much less accurate. This can be assessed via the Dirichlet boundary condition simulations described in section 1 , in which we solved (1) on a large spatial domain with $u=v=0$ imposed at one boundary and $u_{x}=v_{x}=0$ at the other. Provided that $c$ is sufficiently small that the asymptotic wavetrain is stable, the long-term solution has the form of a stationary Nozaki-Bekki hole away from the latter boundary $[51,53]$. A convenient measure of accuracy is provided by the amplitude of the asymptotic wavetrain, given by (2). For all of the computations in this subsection, and also for Figure 1, we used a semi-implicit Crank-Nicolson method, with a uniform grid spacing $\delta x=0.2$ and a time step $\delta t=10^{-3}$; for $c=1.0$, say, the computed asymptotic wavetrain amplitude is then accurate to $0.012 \%$. The implications of this for the computation of sourcesink patterns follows from a consideration of the solution form in between the source and sink, where the two structures interact. The eigenvalues of (5) imply that the difference between the source (respectively, sink) solution and the asymptotic wavetrain is proportional to $e^{-z \sqrt{2}}$ (respectively, $e^{-(L-z) / \sqrt{2}}$ ), where $L$ is the separation distance and $z=0$ is the center of the source. These quantities are the same size when $z \approx L / 3$, with that size being about $e^{-L \sqrt{2} / 3}$. Therefore we expect our PDE simulations to be able to reproduce source-sink patterns with reasonable accuracy, provided that $L$ is less than about $-(3 / \sqrt{2}) \log \left(1.2 \times 10^{-4}\right) \approx 19.5$.

With this restriction in mind, we began by attempting to reproduce in PDE simulations the stationary source-sink pairs corresponding to the various rows of Table 1(a); their typical form is illustrated in Figure 8. We used a relatively large spatial domain (chosen arbitrarily as $0<x<100$ ), with boundary conditions $u_{x}=v_{x}=0$ at both ends. As discussed in section 1 , this condition is compatible with the center of a stationary sink, but it is also satisfied by the (stable) spatially uniform oscillation $u=-\sin (c t), v=\cos (c t)$, which we found to have a very large basin of attraction. Therefore care is required in the choice of initial solution, and we based our initial conditions on our ODE computations of source-sink pairs, described in section 5.1. On the left-hand part of the domain, we used the ODE solution (such as that illustrated in Figure 8), starting from the center of the sink. On the right-hand part of the domain, we again used the ODE solution starting from the center of the sink, but with $z$ decreasing in this case. Finally, in the central part of the domain, we used the asymptotic wavetrain. Continuity of phase, $\theta$, is of course required; we obtained the $\theta$ profile for the source-sink pair by numerically solving $d \theta / d z=\widehat{\psi}$ in parallel with (5), and we then made appropriate uniform shifts in $\theta$ in the central and right-hand parts of the solution, in order to give continuity. We then converted from $r$ and $\theta$ to $u$ and $v$, prior to solving (1). The form of the resulting initial conditions is as in Figure 9(a). Our procedure results in small discontinuities between the three segments, but these disappear after a short time in the simulations.

The significance of the domain being relatively large is that the source is much too far from the sink at the right-hand boundary for there to be any interaction in the PDE simulations with our accuracy level. Thus, up to the cut-off at $x=0$, we are effectively simulating a single, isolated, stationary source-sink pair. Figure $9(\mathrm{~b})$ shows the results of our simulations. We plot the distance $L_{-}$between the sink at $x=0$ and the source (see Figure $9(\mathrm{a})$ ) as a function 

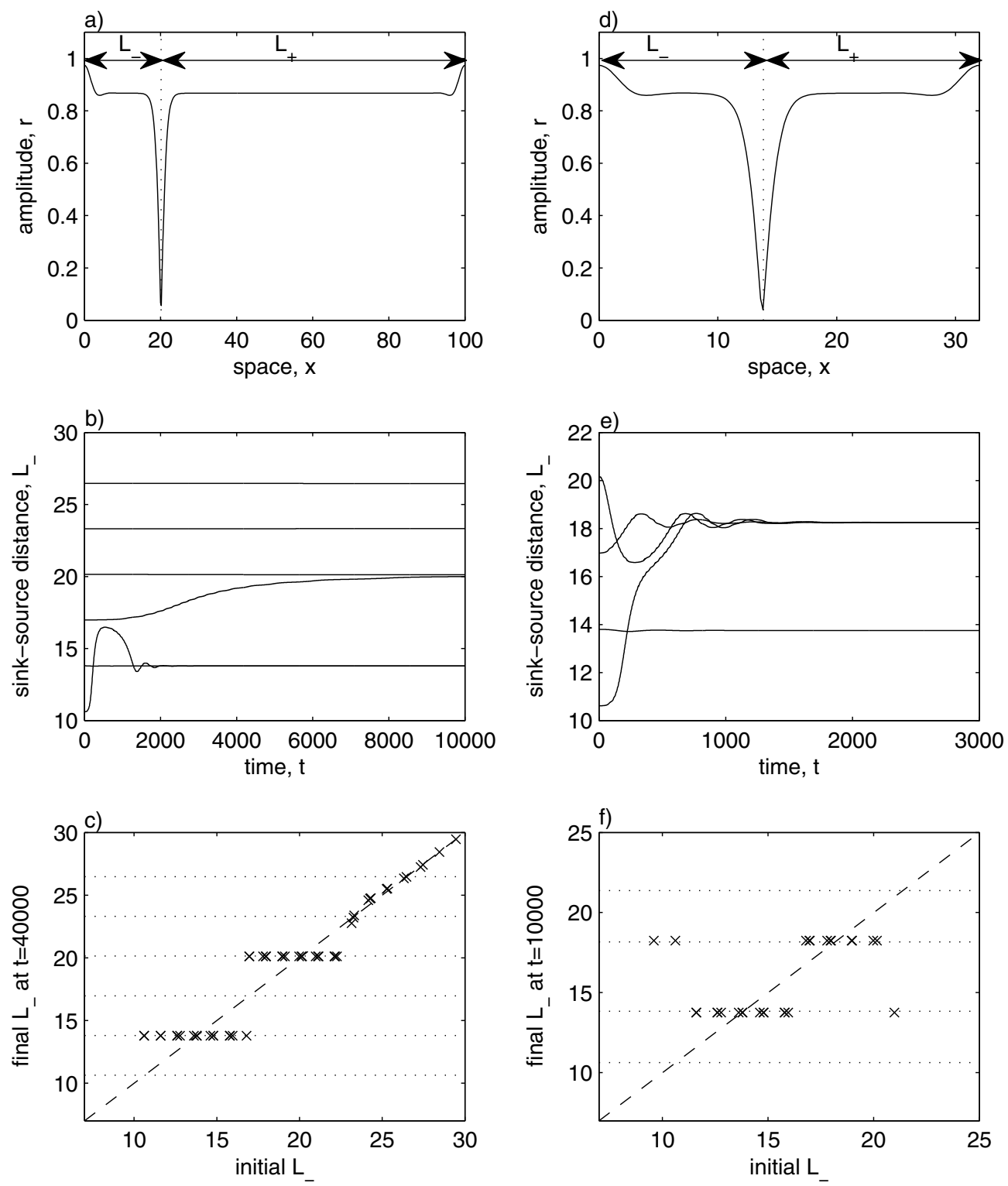

Figure 9. Results of PDE simulations of (1) with $c=1.4$ and boundary conditions $u_{x}=v_{x}=0$ at both boundaries (left boundary at $x=0$ in all simulations, right boundary at $x=100$ in (a)-(c) and at $x=32$ in (d)-(f)). The initial conditions were always a source-sink pair, modified using the methods described in section 5.2. (a), (d) Solutions at time $t=10000$, with source-sink spacing as in our theoretical predictions. (b), (e) Time series of the sink to source distance. (c), (f) The initial sink to source spacing against the final sink to source spacing at the final simulation time $(t=40000$ in $(\mathrm{a})-(\mathrm{c})$ and $t=10000$ in $(\mathrm{d})-(\mathrm{f}))$. In (c) the crosses on the diagonal correspond to cases in which the interactions between the source and the boundary sinks are both dominated by numerical error, as discussed in section 5.2. The equations were solved numerically using a semi-implicit Crank-Nicolson method, with $\delta x=0.2$ and $\delta t=0.001$.

Copyright (c) by SIAM. Unauthorized reproduction of this article is prohibited. 
of time, for each of the initial spacings listed in the first column of Table 1(a). In two cases (initial $L_{-}=13.795$ and 20.136$)$, the spacing stays approximately constant $\left(L_{-}=13.785\right.$ and 20.113 at $t=10^{4}$ ); we attribute the small changes to numerical error. In two other cases (initial $L_{-}=10.624$ and 16.965 ) the spacing changes, converging to about 13.8 and 20.1, respectively. This suggests that the source-sink pairs with these spacings are unstable, with the long time scale on the horizontal axis in Figure 9(b) indicating that the corresponding eigenvalue is very small. Finally, the two largest spacings (initial $L_{-}=23.306$ and 26.476) remain almost constant; there are in fact very slow changes, which are not visible at the scale used in Figure 9(b). In these cases the source-sink separation is large enough that the interaction between the source and sink occurs via variations in the solution amplitude that are below the accuracy of our numerical scheme. To confirm this, we reran a small number of the simulations at higher numerical accuracy $\left(\delta x=0.1, \delta t=10^{-4}\right)$. For initial $L_{-}=23.306$, the separation then converges to about $26.5 .^{2}$

These results show that our boundary and initial conditions are suitable for the simulation of source-sink pairs, providing a foundation from which we can investigate the discreteness of the spacings. For this, we made small changes to the location of the source, keeping the overall domain size constant. For each of the initial spacings used in Figure 9(b), we changed $L_{-}$by $\pm 1, \pm 2, \pm 3$, and \pm 4 , with a corresponding decrease or increase in $L_{+}$. We achieved this by altering the length of the "flat" regions of $r$ and $\theta_{x}$, in which the solution is approximately on the asymptotic wavetrain. Appropriate phase shifts are required in the various parts of the solution in order to retain continuity of phase, prior to conversion from $r$ and $\theta$ to $u$ and $v$. Figure 9(c) shows that in all cases the source-sink spacing converged to either 13.8 or 20.1 (approximately), provided that the initial spacing was sufficiently small to prevent the sourcesink interaction from being dominated by numerical error; we found that the threshold for this is $L_{-} \approx 23$ for our numerical scheme. These results provide specific evidence for the discreteness of possible separations in source-sink pairs.

To study the case of sink-source-sink triples, it was necessary to reduce the domain length considerably, so that the source was close enough to both boundaries for the interactions to be significant within the context of our numerical scheme. We fixed the domain length at 32, for which (40) predicts source-sink separations of $L_{ \pm}=10.625$ and 13.839 , plus their complements. ${ }^{3}$ We generated initial conditions with a variety of different initial spacings, using our numerical solutions of the travelling wave ODEs (5) for source-sink pairs, following the procedure described above. Figure $9(\mathrm{f})$ shows that all of these solutions converged to the same spacing of about $L_{-}=13.75$ (or its complement of 18.25); the difference between this and the theoretical prediction can be attributed to numerical error. The temporal evolution of the separation is shown in Figure 9(e) for a few cases. These results provide evidence that the discreteness of possible separations extends beyond the case of single source-sink pairs to patterns with multiple sources and sinks.

While our PDE tests successfully support our prediction of the discreteness of possible

\footnotetext{
${ }^{2}$ Readers considering reproducing this result should be aware that the separation changes very slowly: a solution time of about $2 \times 10^{5}$ is required to see the convergence.

${ }^{3}$ Equation (40) also has three smaller solutions of about 1.1, 4.3, and 7.5. However, the significance of these is doubtful since the theory underlying (40) assumes large separation distances. We have not seen any of these smaller spacings in our PDE simulations.
}

Copyright ( $)$ by SIAM. Unauthorized reproduction of this article is prohibited. 
separation distances in source-sink patterns, they also highlight that the patterns that emerge in simulations such as those in Figure 1 are the result of a complicated interplay between true source-sink interactions and numerical artifacts arising from insufficient numerical accuracy. For example, Figure 1(g) shows the movement of four source-sink pairs with equal spacings over long simulation times. Our numerical tests in this subsection suggest that the observed distance between each source and the sink on its left $(\approx 14)$ is the result of a real source-sink interaction. However, the distance between the sources and the sinks on their right, which has a range of different values, significantly exceeds the threshold of about 23 in each case, implying that the interactions between the sources and sinks are dominated by numerical error. Further investigation into the determinants of the long-term dynamics of source-sink patterns is a natural (and challenging) area for further investigation. However, a prerequisite for such studies is a numerical scheme that is accurate enough to generate quantitatively correct source-sink solutions for a wide range of parameter values and source-sink spacings.

6. Discussion. Patterns of sources and sinks are a common feature of numerical simulations of the CGLE and have been observed in a variety of corresponding experimental systems. The main results of this paper concern the possible relative locations of sources and sinks that form a coherent pattern moving with constant small speed. Using a formal asymptotic expansion, we showed that the separations $L_{+}$and $L_{-}$between each source and its neighboring sinks are constrained to satisfy a phase locking condition which requires them to lie on one of a discrete infinite sequence of curves in the $L_{+}-L_{-}$plane. Moreover, we have derived exact formulae for the leading order terms in asymptotic expansion of these curves. Our asymptotics are based on two assumptions: large source-sink separations and slow propagation speed. Mathematically, the first assumption ensures that the source resembles a Nozaki-Bekki hole, while the second implies that it is the stationary hole that is relevant. One aspect of our results that is of particular interest is that although the possible separations do depend on the speed at which the sources and sinks move, this dependence tends to zero as the separations tend to infinity. Moreover, our detailed numerical investigation suggests that the dependence of the separations on propagation speed is so small that it is significantly less than the errors inherent in most numerical methods in use for the CGLE (see column 3 of Table 1).

Many readers will be familiar with the "modulated amplitude wave" (Brusch and coworkers [6, 5, 4], Lan, Garnier, and Cvitanović [28]) and "homoclinic hole" (homoclons) (van Hecke [57], van Hecke and Howard [58], Howard and van Hecke [19]) solutions of the CGLE. We now briefly compare these solutions with the source-sink patterns that we have studied. All of these solution types are "coherent structures" satisfying the solution ansatz (4) and the ODEs (5). Briefly, the equilibria of these ODEs, which are wavetrains, can undergo Hopf bifurcation (Janiaud et al. [20]). The branches of periodic solutions (in $\widehat{r}, d \widehat{r} / d z$, and $\widehat{\psi}$ ) emanating from such bifurcations constitute the modulated amplitude waves. In some cases, the solution branch terminates at another Hopf bifurcation; otherwise the period is unbounded along the branch, and the terminating homoclinic orbit is known as a homoclinic hole. The case of a single source-sink pair that we have studied is superficially similar to a homoclinic hole: both are localized disturbances to an otherwise uniform wavetrain solution. However, homoclinic holes do not contain a band of an oppositely moving wavetrain. Moreover, homoclinic holes are connected to a Hopf bifurcation of an equilibrium of (5) (a wavetrain), while

Copyright (c) by SIAM. Unauthorized reproduction of this article is prohibited. 
source-sink pairs are not-rather, their organizing center is a heteroclinic cycle. Similarly, modulated amplitude waves are quite distinct from repeating patterns of sources and sinks.

The focus of this paper has been the existence of source-sink patterns. An important follow-up question is the stability of these patterns. As discussed in section 2, patterns of sources and sinks provide a classic illustration of the difference between convective and absolute stability. When the asymptotic wavetrains are only convectively unstable, all unstable linear modes propagate as they grow, travelling towards the nearest sink, where they are absorbed. In contrast, when one of the asymptotic wavetrains is absolutely unstable, there are stationary unstable modes. Therefore, one expects intuitively that in the limit of large separations, the stability of a solution consisting of a doubly infinite sequence of sources and sinks will depend crucially on the absolute stability of the asymptotic wavetrain but not on its stability. This expectation is consistent with the significant body of work on the highly analogous problem of pulse stability (Sandstede and Scheel [48], Nii [33], Romeo and Jones [42]). These various papers consider pulse wave solutions of reaction-diffusion equations, with a stable uniform equilibrium behind and ahead of the wave, and a long intermediate plateau in which the solution is close to a second equilibrium. In particular, [48] showed that a solution of this type is stable in the limit of large plateau lengths if and only if two conditions are satisfied: (i) the plateau equilibrium is absolutely stable, and (ii) a finite set of point eigenvalues associated with the front and back of the pulse are all stable. The intuition behind (i) is exactly as discussed above: provided that the plateau equilibrium is absolutely stable, any unstable linear mode will propagate as it grows, eventually reaching the front or back of the pulse, where it will be absorbed.

Condition (ii) reflects the fact that the front and back of the pulse may themselves be intrinsically unstable, through point spectra. Sandstede and Scheel [48] showed that such point spectra are inherited by the pulse, with a correction that is exponentially small in the separation distance. For the source-sink patterns that we are considering, the corresponding issue is point spectra associated with the sources and sinks. For sinks, the only detailed studies that we are aware of are restricted to either $c \ll 1$ (Kapitula [21]), or to "weak shocks," in which the wavenumbers of the two asymptotic wavetrains are very similar (Kapitula [22]). Neither of these cases is relevant to the present study, but a large body of PDE simulations suggests that the point spectra associated with sinks are always stable. However, this is certainly not the case for sources, and the stability of Nozaki-Bekki holes has been studied in considerable detail (Sakaguchi [43], Chaté and Manneville [9], Sasa and Iwamoto [49], Popp et al. [40], Kapitula and Rubin [23]; a concise review of this work can be found in [29]; see also Sandstede and Scheel [45]). In particular, the stability of stationary Nozaki-Bekki holes changes due to discrete eigenvalues as parameters cross the "core stability curve" in the parameter plane of the CGLE. By analogy with the results of Sandstede and Scheel [48], we hypothesize that these discrete eigenvalues will be inherited by the source-sink patterns, modulo a correction that is exponentially small in the source-sink separation. The core stability curve crosses the zero linear dispersion axis at $c=c^{\text {core }} \approx 1.12$, slightly above the threshold for the stability of the asymptotic wavetrains $\left(c=c^{\text {stab }} \approx 1.11\right) .{ }^{4}$ Therefore we hypothesize that patterns

\footnotetext{
${ }^{4}$ The difference between these two critical values of $c$ is somewhat ambiguous in the literature, and we are grateful to Professor Shin-ichi Sasa (University of Tokyo) for confirming it in personal correspondence.
}

Copyright (C) by SIAM. Unauthorized reproduction of this article is prohibited. 
consisting of a doubly infinite sequence of sources and sinks can be stable, in the limit of large separations, only if $c$ lies between $c^{\text {core }} \approx 1.12$ and $c^{\text {abs }} \approx 1.57$. Since rigorous results already exist for the reaction-diffusion pulse problem, a proof of this hypothesis seems within reach and is a natural area for future study.

In the range $c^{\text {core }}<c<c^{\text {abs }}$, we expect that stability depends on eigenvalues near the origin. By analogy with multipulse solutions (Sandstede [44]), each source and sink is expected to contribute one such eigenvalue. These are associated with the neutral translation modes of the individual sources and sinks, but only one will be pinned at the origin as the translation mode of the entire pattern. This situation differs from that of pulse waves discussed above; in that case the plateau and background states have different stabilities, and as a result only the "back" of the pulse generates an eigenvalue associated with its neutral translation mode (see Lemma 3 of [48]).

As a final remark on stability, we comment that the above discussion concerns only a doubly infinite sequence of sources and sinks. It is unchanged for a source-sink pattern on a finite domain with either periodic or appropriate separated boundary conditions. It is also unchanged for a finite or singly infinite sequence of sources and sinks on an unbounded domain, when the sequence is terminated by $\operatorname{sink}(\mathrm{s})$. However, in the case of a terminal source, even propagating unstable modes can grow without bound, since the group velocity is then directed away from the source, into an unbounded part of the domain containing no sinks. Therefore it is the stability of the asymptotic wavetrain, rather than its absolute stability, that is relevant in this case.

In addition to issues connected to the stability of source-sink patterns, our results raise four main questions that are natural targets for future work:

1. In our perturbation theory calculation, we considered only the leading order difference between a source-sink pattern and the corresponding isolated sources and sinks. This was sufficient to provide a detailed account of the separation between a source and its neighboring $\operatorname{sink}(\mathrm{s})$. However, neighboring sinks are decoupled at this order, and thus our calculation provides no information on the nature or even existence of constraints on sink-sink separations. Investigation of this via higher order asymptotics is an important objective for subsequent work.

2. Our results apply only to the case of zero linear dispersion, and an obvious question is the extent to which our results apply when the linear dispersion parameter in the CGLE is nonzero. The cornerstone of our calculation was the exact solution for the stationary isolated source. The work of Nozaki and Bekki [34] provides corresponding exact solutions for nonzero linear dispersion, so that a directly analogous calculation would be possible, with the only barrier being the increased algebraic complexity.

3. Generically, sources occur as isolated solutions in reaction-diffusion-type equations (van Saarloos and Hohenberg [60], Sandstede and Scheel [46]). However, the CGLE is nongeneric and has a continuous family of sources, the Nozaki-Bekki holes. The "hidden symmetry" that is responsible for this has been discussed extensively (van Saarloos and Hohenberg [60], Doelman [14], Lega [29]). We have focussed entirely on the case of slowly moving source-sink patterns with large separation distances, so that the sources are close to the stationary Nozaki-Bekki hole solution. It seems likely that there are also more rapidly moving source-sink patterns, in which the sources are close to the 
corresponding nonstationary Nozaki-Bekki holes. Such patterns could also be studied using our perturbation theory approach, although the greater algebraic complexity of the moving Nozaki-Bekki hole solutions would make the calculation extremely challenging. We emphasize that the existence of such patterns would be nongeneric and would again be a consequence of the hidden symmetry of the CGLE. Moreover, all nonstationary Nozaki-Bekki holes are structurally unstable; in particular, they do not persist when the equation is perturbed by the addition of a small quintic term (Doelman [14], Popp and coworkers [41, 40]). We speculate that this property will be shared by moving source-sink patterns, including the solutions that we have studied with $s_{1} \neq 0$.

4. We have shown that there is a family of source-sink patterns, with a discrete set of possible source-sink separations and a continuum of possible propagation speeds. Whenever a source-sink pattern arises in a solution of the CGLE, a particular member of this family is selected by the initial and boundary conditions. This pattern selection problem is completely open.

Appendix A. In this appendix, we give details of the various constants arising in the perturbation theory calculation for which we have exact formulae. All depend on the amplitude $R^{*}$ of the isolated source, which is equivalent to a dependence on the parameter $c$, via (13).

$$
\begin{aligned}
& H_{1}=4 \sqrt{2}\left(\frac{R^{*}}{W}\right) \sqrt{1-R^{* 2}} \operatorname{Re} \int_{0}^{1}\left[F\left(\alpha, \beta, \gamma ; \frac{1+t}{2}\right)-F\left(\alpha, \beta, \gamma ; \frac{1-t}{2}\right)\right] t^{2}\left(1-t^{2}\right)^{(-1+i \delta) / 2} d t, \\
& H_{2}=2 \sqrt{2}\left(\frac{R^{*}}{W}\right) \operatorname{Re}\left\{\int_{0}^{1} F\left(\alpha, \beta, \gamma ; \frac{1+t}{2}\right) \cdot\left[\left(3-2 R^{* 2}\right) t^{3}-t\right]\left(1-t^{2}\right)^{(-1+i \delta) / 2} d t\right. \\
& \left.-\int_{0}^{1} F\left(\alpha, \beta, \gamma ; \frac{1-t}{2}\right) \cdot\left[\left(3-2 R^{* 2}\right) t^{3}-t\right]\left(1-t^{2}\right)^{(-1+i \delta) / 2} d t\right\}, \\
& I_{1}=4 \sqrt{2}\left(\frac{R^{*}}{W}\right) \sqrt{1-R^{* 2}} \operatorname{Re} \int_{0}^{1}\left[F\left(\alpha, \beta, \gamma ; \frac{1+t}{2}\right)+F\left(\alpha, \beta, \gamma ; \frac{1-t}{2}\right)\right] t^{2}\left(1-t^{2}\right)^{(-1+i \delta) / 2} d t, \\
& I_{2}=2 \sqrt{2}\left(\frac{R^{*}}{W}\right) \operatorname{Re} \int_{0}^{1}\left[F\left(\alpha, \beta, \gamma ; \frac{1+t}{2}\right)+F\left(\alpha, \beta, \gamma ; \frac{1-t}{2}\right)\right] \\
& \cdot\left[\left(3-2 R^{* 2}\right) t^{3}-t\right]\left(1-t^{2}\right)^{(-1+i \delta) / 2} d t \\
& Q_{+}=\frac{i \delta}{3+i \delta}\left[\frac{2^{-1+i \delta} \Gamma(i \delta)^{2}}{\Gamma\left(\frac{1}{2}+i \delta+i\left\{\delta^{2}+\frac{3}{4}\right\}^{1 / 2}\right) \Gamma\left(\frac{1}{2}+i \delta-i\left\{\delta^{2}+\frac{3}{4}\right\}^{1 / 2}\right)}\right. \\
& \left.-\frac{2^{-1-i \delta}|\Gamma(i \delta)|^{2}}{\left|\Gamma\left(\frac{1}{2}+i\left\{\delta^{2}+\frac{3}{4}\right\}^{1 / 2}\right)\right|^{2}}\right], \\
& Q_{-}=\frac{-2^{-1-i \delta}}{3+i \delta} \\
& C_{1}^{\xi}=\kappa_{\xi} \operatorname{sign}(\epsilon) \operatorname{Im}\left[\bar{Q}_{-\xi} S \exp \left\{-i \delta\left(\log |\epsilon|+\log \kappa_{\xi}\right)\right\}\right] / \operatorname{Im}\left[Q_{\xi} \bar{Q}_{-\xi}\right]+o(1), \\
& C_{2}^{\xi}=\kappa_{\xi} \operatorname{sign}(\epsilon) \operatorname{Im}\left[\bar{Q}_{\xi} S \exp \left\{-i \delta\left(\log |\epsilon|+\log \kappa_{\xi}\right)\right\}\right] / \operatorname{Im}\left[Q_{\xi} \bar{Q}_{-\xi}\right]+o(1) .
\end{aligned}
$$

When evaluating these various expressions numerically, we used the software package MAPLE (Monagan et al. [30]); our code is listed in 78096_01.zip [82.2KB]. 
Proof that $\boldsymbol{H}_{\mathbf{2}}=0$. Substituting $\xi=(1+t) / 2$ into the first of the integrals in (A.1) and $\xi=(1-t) / 2$ into the second gives

$$
\begin{aligned}
H_{2} & =2^{(5 / 2)+i \delta}\left(R^{*} / W\right) \operatorname{Re}\left[\left(4 R^{* 2}-6\right) h_{1}+\left(1-R^{* 2}\right) h_{-1}\right], \\
\text { where } \quad h_{n} & =\int_{0}^{1}\left(\xi-\xi^{2}\right)^{(n+i \delta) / 2}(2 \xi-1) F(\alpha, \beta, \gamma ; \xi) d \xi .
\end{aligned}
$$

Now $F($.$) satisfies the hypergeometric equation F(\alpha, \beta, \gamma ; \xi)=\left(\xi-\xi^{2}\right) \cdot\left(d^{2} F / d \xi^{2}\right) /(1+i \delta)-$ $(2 \xi-1) \cdot(d F / d \xi)$. Therefore

$$
h_{1}=\frac{1}{1+i \delta} \int_{0}^{1}\left(\xi-\xi^{2}\right)^{(3+i \delta) / 2}(2 \xi-1) \frac{d^{2} F}{d \xi^{2}} d \xi-\int_{0}^{1}\left(\xi-\xi^{2}\right)^{(1+i \delta) / 2}(2 \xi-1)^{2} \frac{d F}{d \xi} d \xi .
$$

Evaluating the first of these integrals by parts implies

$$
h_{1}=\int_{0}^{1}\left\{\frac{(1-i \delta)^{2}}{2\left(1+\delta^{2}\right)}\left(\xi-\xi^{2}\right)^{(1+i \delta) / 2}(2 \xi-1)^{2}-\frac{2(1-i \delta)}{1+\delta^{2}}\left(\xi-\xi^{2}\right)^{(3+i \delta) / 2}(2 \xi-1)\right\} \frac{d F}{d \xi} d \xi .
$$

Evaluating by parts again and simplifying gives $\left(28+4 \delta^{2}\right) h_{1}=\left(1+\delta^{2}\right) h_{-1}$. Using $\delta^{2}=$ $11-12 R^{* 2}$, this implies that $H_{2}=0$.

Appendix B. The lack of an exact solution for sinks means that we do not have a formula for the constant $S$, which must therefore be calculated numerically. An isolated stationary sink is a solution of the ODEs (5), with $s=0$ and $d \widehat{\theta}(t) / d t=K_{0}$, defined in (10). As $z \rightarrow-\infty$ (say), such a sink solution must approach the steady state $\widehat{r}=R^{*}, \widehat{\psi}=-\sqrt{1-R^{* 2}}$ on the two-dimensional manifold corresponding to the complex conjugate eigenvalues $(1 \pm i \delta) / \sqrt{2}$. Therefore calculation of the sink solution can be reduced to a shooting problem. We solve (5), with $s=0$ and $d \widehat{\theta}(t) / d t=K_{0}$, forwards in $z$ from initial conditions

$$
(\widehat{r}, d \widehat{r} / d z, \widehat{\psi})=\left(R^{*}, 0,-\sqrt{1-R^{* 2}}\right)+\rho \operatorname{Re}\left(\underline{E} \mathrm{e}^{i \sigma}\right)
$$

at $z=0$ (say). Here $\underline{E}$ is the eigenvector corresponding to the eigenvalue $(1+i \delta) / \sqrt{2}$, normalized so that $E_{1}=1$. The quantities $\rho$ (small) and $\sigma \in[0,2 \pi)$ are the polar coordinates of the starting point on the unstable eigenspace, which is used as an approximation of the unstable manifold. Our shooting parameter is $\sigma$, and the choice of $\rho$ is discussed below. We integrate forwards in $z$, detecting zeros of $d \widehat{r} / d z$ and $\widehat{\psi}$ as the integration proceeds. For the numerical solution, we used the routine DLSODAR (Hindmarsh [18], Petzold [38]), which is part of the ODEPACK collection. This solver automatically switches between an Adams predictor-corrector method and a Gear backward differentiation formula method, and automatically stops integration when specified algebraic conditions are satisfied. This root-finding capability enables accurate detection of the zeros of $d \widehat{r} / d z$ and $\widehat{\psi}$. The numerical code is freely available at http://www.netlib.org.

A sink occurs when $d \widehat{r} / d z$ and $\widehat{\psi}$ are simultaneously zero; this is then the center of the sink, about which the sink solution is symmetric. For all values of $c$ that we have considered, this occurs for exactly one value of $\sigma$. This is in line with the generic expectation of exactly one sink solution for any given pair of asymptotic wavetrain amplitudes [60]. However, we emphasize

Copyright (C) by SIAM. Unauthorized reproduction of this article is prohibited. 
that a proof of this is currently lacking, to the best of our knowledge. The critical value $\sigma_{\text {crit }}$ can be calculated using a nonlinear algebraic equation solver in which the "equation" is the (signed) distance between the relevant zeros of $d \widehat{r} / d z$ and $\widehat{\psi}$; we denote by $z_{\text {crit }}$ the coordinate of the common zero. Having found $\sigma_{\text {crit }}$ and $z_{\text {crit }}$, we can deduce the value of $S$, which is defined by (31). Comparison with (B.1) shows that we require

$$
\rho \operatorname{Re}\left[\mathrm{e}^{i \sigma_{c r i t}}\right]=\operatorname{Re}\left[S \mathrm{e}^{-z_{c r i t}(1+i \delta) / \sqrt{2}}\right],
$$

which implies that $|S|=\rho \mathrm{e}^{z_{\text {crit }} / \sqrt{2}}$ and $\arg (S)=\delta z_{\text {crit }} / \sqrt{2} \pm \sigma_{\text {crit }}$. The choice of $\pm \sigma_{\text {crit }}$ is specified by the additional requirement of correspondence in $d r / d z$ between (31) and (B.1), which implies that $\arg (S)=\delta z_{\text {crit }} / \sqrt{2}+\sigma_{\text {crit }}$.

The above algorithm requires a particular value for the (small) parameter $\rho$. In the numerical ODE solver DLSODAR, errors are controlled locally, and we denote the local error tolerance by $\ell$. In all our implementations of this solver, we set numerical parameters so that the local error test during the solution is met if either the absolute or relative error is less than $\ell$, for each solution component. Then after a few numerical integration steps, the initial conditions (B.1) evolve to

$$
(r, d r / d z, \psi)=\left(R^{*}, 0,-\sqrt{1-R^{* 2}}\right)+\rho \operatorname{Re}\left(\underline{E} \mathrm{e}^{i \sigma} \mathrm{e}^{(1+i \delta) z / \sqrt{2}}\right)+\ell \underline{\mathcal{L}}(z)+\rho^{2} \underline{\mathcal{R}}
$$

for some small positive value of $z ; \underline{\mathcal{L}}$ and $\underline{\mathcal{R}}$ are $O(1)$ as $\ell$ and $\rho \rightarrow 0$. The $O\left(\rho^{2}\right)$ term arises from the nonlinear terms in the ODEs. The deviation of this point from the eigenvector is given by

$$
\frac{\rho \operatorname{Re}\left(E_{m} \mathrm{e}^{i \sigma} \mathrm{e}^{(1+i \delta) z / \sqrt{2}}\right)+\ell \mathcal{L}_{m}(z)+\rho^{2} \mathcal{R}_{m}}{\rho \operatorname{Re}\left(E_{n} \mathrm{e}^{i \sigma} \mathrm{e}^{(1+i \delta) z / \sqrt{2}}\right)+\ell \mathcal{L}_{n}(z)+\rho^{2} \mathcal{R}_{n}}-\frac{\operatorname{Re}\left(E_{m} \mathrm{e}^{i \sigma}\right)}{\operatorname{Re}\left(E_{n} \mathrm{e}^{i \sigma}\right)}
$$

$(m, n \in\{1,2,3\}, m \neq n)$, which is $O(\rho+\ell / \rho)$ as $\ell, \rho \rightarrow 0$. The optimal choice for $\rho$ in this setting is that which minimizes this deviation, namely that $\rho$ is proportional to $\sqrt{\ell}$. With this choice, the deviation from the eigenvector is $O(\sqrt{\ell})$ as $\ell \rightarrow 0$. Since the third eigenvalue at the steady state is (real and) negative, the solution returns to the required trajectory as $z$ increases. However, the deviation from the eigenvector at the start of the integration leads to an $O(\sqrt{\ell})$ error in the value of $z$ corresponding to a particular point $(r, d r / d z, \psi)$ on this trajectory. Since the governing equations are autonomous, this $O(\sqrt{\ell})$ translational error approaches a constant, independent of the point on the solution trajectory.

In 78096_01.zip [82.2KB], we describe a test problem that corroborates this argument. It follows that the numerical error in $z_{\text {crit }}$ will be $O(\sqrt{\ell})$. To consider the numerical error in $\sigma_{\text {crit }}$, we compared the convergence of $z_{\text {crit }}$ and $\sigma_{\text {crit }}$ as $\ell$ is decreased; we fix $\rho$ rather than setting $\rho=\sqrt{\ell}$, since the value of $\sigma_{\text {crit }}$ (and of $z_{\text {crit }}$ ) depends on $\rho$. This comparison suggests that $z_{\text {crit }}$ and $\sigma_{\text {crit }}$ converge in parallel, suggesting that the error in both quantities, and hence in both the modulus and argument of $S$, will be $O(\sqrt{\ell})$. We take $\ell=10^{-24}$, using 16-byte precision, which gives about 34 significant digits. We then expect the error in $S$ to be about $10^{-12}$, giving us a high level of confidence in the first 10 or 11 decimal places of our computed values of $S$. A table of $S$ values as a function of $c$, to 10 decimal places, is given in 78096_01.zip $[82.2 \mathrm{~KB}]$. 
Appendix C. In this appendix we discuss the numerical errors in the entries in Table 1. Since the constant $S$ must be calculated numerically, there are numerical errors in the predicted values listed in the table, as well as in the simulated values. Hence the predictions are the sum of the true values, numerical error, and corrections due to higher order terms in the asymptotic expansions. Here we justify our assertion that in all three cases (separation, $\epsilon$, and spike height), the numerical errors in both predicted and simulated values are significantly less than the difference between the two values. It follows from this that the differences (which are listed in columns 3,4 , and 6 of Table 1) are an accurate measure of the higher order corrections.

We denote by $\mathcal{E}_{q}$ the numerical error in a quantity $q$. Then our algorithm for calculating $S$ (see Appendix B) implies that to leading order for small errors, $\mathcal{E}_{|S|}=|S| \mathcal{E}_{z_{\text {crit }}} / \sqrt{2}$ and $\mathcal{E}_{\arg (S)}=(\delta / \sqrt{2}) \mathcal{E}_{z_{c r i t}}+\mathcal{E}_{\sigma_{\text {crit }}}$. The results in Table 1 are for an isolated source-sink pair, for which our perturbation theory calculation implies that the source-sink separation $L$ is one of the values given by (41), with the corresponding value of $\epsilon$ given by (43). The numerical errors in our evaluations of these predictions enter via $\widetilde{\Upsilon}$, which depends on $S$. Straightforward expansions show that

$$
\begin{aligned}
\mathcal{E}_{L} & =\mathcal{E}_{z_{\text {crit }}}+(\sqrt{2} / \delta) \mathcal{E}_{\sigma_{\text {crit }}}, \\
\mathcal{E}_{\epsilon} & =(\sqrt{2} / \delta)|\epsilon| \mathcal{E}_{\sigma_{\text {crit }}} .
\end{aligned}
$$

Crucially, the errors in $z_{\text {crit }}$ and $\sigma_{\text {crit }}$ translate into absolute errors in $L$, but only relative errors in $\epsilon$. As discussed in Appendix B, our choice of local error tolerance $\left(\ell=10^{-24}\right)$ implies that $\mathcal{E}_{z_{c r i t}}$ and $\mathcal{E}_{\sigma_{c r i t}}$ are both about $10^{-12}$. Therefore we are confident in our evaluations of the first 10 or 11 decimal places of $L$, and the first 10 or 11 significant figures of $\epsilon$. Hence the numerical errors are significantly less than the differences between the predicted and simulated values in all rows of Table 1, as required. Note, however, that for the next separation in the sequence $(L \approx 29.6)$, the higher order terms in the asymptotic expansion for $L$ would be only about 100 times larger than the numerical error in the computation.

The predicted height of the spike in $\widehat{\psi}$ is given by (44); it is independent of $S$ and hence free from numerical error. In numerical solutions of (5), the error in the height depends on $\ell$ and $\epsilon$. Recall that we set numerical parameters in DLSODAR so that the local error test during ODE solution is met if either the absolute or relative error is less than $\ell$ for each solution component. Since the spike in $\psi$ has height $O(1 / \epsilon)$, the absolute local error near its peak is $O(\ell / \epsilon)$. Moreover, one expects the number of solution steps required to reach the top of the spike to be $O(1 / \epsilon)$, suggesting a global error estimate of $O\left(\ell / \epsilon^{2}\right)$. A test problem corroborating this is described in 78096_01.zip [82.2KB]. Note that this rather large global error does not persist as integration of (5) continues beyond the spike. This is due to the structure of the transition layer equations (20) which determine the spike. The exact solution (21a), (21b) of (20) shows that numerical errors in $\psi$ propagate forward only as relative rather than absolute error, so that the residual global error is only $O(\ell / \epsilon)$.

It remains to consider the numerical errors in the values of $\epsilon$ and source-sink separation that we have determined by numerical solution of the ODEs (5). These are difficult to estimate because they involve a complex mixture of different numerical errors, and there is no natural analogous problem with an exact solution. Moreover, the steep spike in $\psi$ (see Figure 8)

Copyright (c) by SIAM. Unauthorized reproduction of this article is prohibited. 
makes an adaptive ODE solver essential, and this makes convergence acceleration techniques for error estimation inappropriate, because of potentially nonlinear changes in step sizes as the local error is decreased. To give a rough indication of the errors, we calculated the variation in the computed values as the local error tolerance $\ell$ was changed over five orders of magnitude $\left(10^{-21}-10^{-25}\right)$ spanning our reference value $\left(10^{-24}\right)$, for each of the rows in Table 1 . At worst, the source-sink separation and the value of $\epsilon$ change in only the 12th and 20th decimal places, respectively. This suggests that the numerical errors in these computed values are comparable with those in our evaluations of the analytical predictions, and significantly less than the differences between the computed and predicted values.

As a final check, we repeated all the calculations in Table 1 (for both the predicted and computed values) at a 100 -fold larger local error tolerance $\left(\ell=10^{-22}\right)$. The entries were all unchanged.

Appendix D. In this appendix we prove that the spirals $\mathcal{S}_{ \pm}$, which are defined as the two sides of (38), have only a finite number of intersections, unless the centers of the spirals lie on one another. Specifically, we prove the following claim.

Proposition. For fixed $\epsilon$, (38) has a finite number of positive solutions for $\kappa_{ \pm}$unless $\Upsilon$ satisfies (37).

In our proof, we make use of the following lemma.

Lemma. If $\kappa_{ \pm} \geq 0$ solves (38) for some $\epsilon$, then $\kappa_{ \pm} \leq \kappa_{\mathrm{m}}=|\Upsilon| /\left(1-\mathrm{e}^{-\pi / 2 \delta}\right)$.

We comment that $|\Upsilon|$ and thus $\kappa_{\mathrm{m}}$ are independent of $\epsilon$.

Proof of the proposition. Eliminating $\kappa_{-}$(say) from (38) yields, to leading order,

$$
g\left(\kappa_{+}\right):=\arg \left(\Upsilon-\kappa_{+} \mathrm{e}^{i \delta \log \kappa_{+}}\right)-\delta \log \left|\Upsilon-\kappa_{+} \mathrm{e}^{i \delta \log \kappa_{+}}\right|=0 .
$$

The lemma implies that $\kappa_{+}$is bounded, so that an infinite number of solutions would accumulate at some $\kappa_{0}$. We will show that this is not possible.

The case $\Upsilon=\kappa_{0} \mathrm{e}^{i \delta \log \kappa_{0}}$, which is equivalent to (37), is excluded by assumption. Hence, $\kappa_{0}$ is such that $g(\kappa)$ is analytic in a neighborhood of $\kappa_{0}$ (on the covering space of arg). Therefore, accumulation of roots at $\kappa_{0}$ implies $g(\kappa) \equiv 0$ in a neighborhood of $\kappa_{0}$. By analytic continuation this holds globally for $\kappa>0$, which would imply that the spirals coincide. However, by the lemma, the spirals are disjoint for large $\kappa_{ \pm}$.

Proof of the lemma. For notational simplicity, we write

$$
\widehat{\kappa}_{+}=\frac{\kappa_{+}}{\mathrm{e}^{(\arg \Upsilon) / \delta}}, \quad \widehat{\kappa}_{-}=\frac{\kappa_{-}}{\mathrm{e}^{(\arg \Upsilon) / \delta}}, \quad A=\frac{|\Upsilon|}{\mathrm{e}^{(\arg \Upsilon) / \delta}},
$$

which reduces $(38)$ to

$$
\widehat{\kappa}_{+} \mathrm{e}^{i \delta \log \widehat{\kappa}_{+}}+\widehat{\kappa}_{-} \mathrm{e}^{i \delta \log \widehat{\kappa}_{-}}=A .
$$

Since the statement is trivial for $\kappa_{+}=0$ or $\kappa_{-}=0$, we assume that $\widehat{\kappa}_{+} \geq \widehat{\kappa}_{-}>0$. Now suppose $\kappa_{+}>\kappa_{\mathrm{m}}$, which is equivalent to $\widehat{\kappa}_{+}>A /\left(1-\mathrm{e}^{-\pi / 2 \delta}\right)$. Then

$$
\begin{aligned}
& \widehat{\kappa}_{-}=\left|A-\widehat{\kappa}_{+} \mathrm{e}^{i \delta \log \widehat{\kappa}_{+}}\right| \geq \widehat{\kappa}_{+}-A \\
& \Rightarrow 1 \geq \widehat{\kappa}_{-} / \widehat{\kappa}_{+} \geq 1-A / \widehat{\kappa}_{+} \geq \mathrm{e}^{-\pi / 2 \delta} \\
& \Rightarrow 1 \geq \cos \left[\delta \log \left(\widehat{\kappa}_{-} / \widehat{\kappa}_{+}\right)\right] \geq 0 .
\end{aligned}
$$

Copyright $\odot$ by SIAM. Unauthorized reproduction of this article is prohibited. 
Now considering (D.2), we have

$$
\begin{aligned}
\left|\widehat{\kappa}_{+} \mathrm{e}^{i \delta \log \widehat{\kappa}_{+}}+\widehat{\kappa}_{-} \mathrm{e}^{i \delta \log \widehat{\kappa}_{-}}\right| & =\left|\widehat{\kappa}_{+}+\widehat{\kappa}_{-} \mathrm{e}^{i \delta \log \left(\widehat{\kappa}_{-} / \widehat{\kappa}_{+}\right)}\right| \\
& \geq\left|\widehat{\kappa}_{+}+\widehat{\kappa}_{-} \cos \left[i \delta \log \left(\widehat{\kappa}_{-} / \widehat{\kappa}_{+}\right)\right]\right| \\
& =\widehat{\kappa}_{+}+\widehat{\kappa}_{-} \cos \left[i \delta \log \left(\widehat{\kappa}_{-} / \widehat{\kappa}_{+}\right)\right] \quad \text { using (D.3) } \\
& \geq \widehat{\kappa}_{+}>A .
\end{aligned}
$$

This is a contradiction of (D.2).

Acknowledgments. We thank Wim van Saarloos (Universiteit Leiden), Björn Sandstede (Brown University), and Shin-ichi Sasa (University of Tokyo) for helpful discussions. JAS thanks Lubomir Banas, Dugald Duncan, Andrew Lacey, and Gabriel Lord (all Heriot-Watt University) for advice. JAS also thanks Dorothy Sherratt for her interest in this subject. MJS thanks the MSRC Tools and Technology Group (Computational Science) for technical assistance.

\section{REFERENCES}

[1] R. Alvarez, M. van Hecke, and W. van SaArloos, Sources and sinks separating domains of left-and right-traveling waves: Experiment versus amplitude equations, Phys. Rev. E, 56 (1997), pp. R1306R1309.

[2] I. S. Aranson and L. Kramer, The world of the complex Ginzburg-Landau equation, Rev. Modern Phys., 74 (2002), pp. 99-143.

[3] T. Bohr, G. Huber, AND E. OTT, The structure of spiral-domain patterns and shocks in the $2 D$ complex Ginzburg-Landau equation, Phys. D, 106 (1997), pp. 95-112.

[4] L. Brusch, A. Torcini, And M. BÄr, Nonlinear analysis of the Eckhaus instability: Modulated amplitude waves and phase chaos with nonzero average phase gradient, Phys. D, 174 (2003), pp. 152-167.

[5] L. Brusch, A. Torcini, M. van Hecke, M. G. Zimmermann, And M. Bär, Modulated amplitude waves and defect formation in the one-dimensional complex Ginzburg-Landau equation, Phys. D, 160 (2001), pp. 127-148.

[6] L. Brusch, M. G. Zimmermann, M. van Hecke, M. Bär, and A. Torcini, Modulated amplitude waves and the transition from phase to defect chaos, Phys. Rev. Lett., 85 (2000), pp. 86-89.

[7] J. Burguete, H. Chaté, F. Daviaud, And N. Mukolobwiez, Bekki-Nozaki amplitude holes in hydrothermal nonlinear waves, Phys. Rev. Lett., 82 (1999), article 3252.

[8] H. Chaté, Spatiotemporal intermittency regimes of the one-dimensional complex Ginzburg-Landau equation, Nonlinearity, 7 (1994), pp. 185-204.

[9] H. Chaté And P. Manneville, Stability of the Bekki-Nozaki hole solutions to the one-dimensional complex Ginzburg-Landau equation, Phys. Lett. A, 171 (1992), pp. 183-188.

[10] J. Cisternas And O. Descalzi, Sources and sinks in the vicinity of a weakly inverted instability, Internat. J. Bifur. Chaos Appl. Sci. Engrg., 17 (2007), pp. 2821-2826.

[11] E. J. Doedel, Nonlinear numerics, J. Franklin Inst., 334 (1997), pp. 1049-1073.

[12] E. J. Doedel, AUTO, A program for the automatic bifurcation analysis of autonomous systems, Congr. Numer., 30 (1981), pp. 265-384.

[13] E. J. Doedel, W. Govaerts, Y. A. Kuznetsov, And A. Dhooge, Numerical continuation of branch points of equilibria and periodic orbits, in Modelling and Computation in Dynamical Systems, E. J. Doedel, G. Domokos, and I. G. Kevrekidis, eds., World Scientific, River Edge, NJ, 2006, pp. 145-164.

[14] A. Doelman, Breaking the hidden symmetry in the Ginzburg-Landau equation, Phys. D, 97 (1996), pp. $398-428$.

[15] G. B. Ermentrout, X. Chen, And Z. Chen, Transition fronts and localized structures in bistable reaction-diffusion equations, Phys. D, 108 (1997), pp. 147-167.

Copyright $\odot$ by SIAM. Unauthorized reproduction of this article is prohibited. 
[16] P. Habdas And J. R. DE BRuyn, Dynamics of defects and travelling waves in an interfacial finger pattern, Phys. D, 200 (2005), pp. 273-286.

[17] P. Habdas, M. J. CASE, AND J. R. DE BRuYn, Behavior of sink and source defects in a one-dimensional traveling finger pattern, Phys. Rev. E, 63 (2001), article 066305.

[18] A. C. Hindmarsh, ODEPACK: A systematized collection of ODE solvers, in Scientific Computing, R. S. Stepleman, M. Carver, R. Peskin, W. F. Ames, and R. Vichnevetsky, eds., North-Holland, Amsterdam, 1983, pp. 55-64.

[19] M. HowARD AND M. VAN HeCKe, Hole-defect chaos in the one-dimensional complex Ginzburg-Landau equation, Phys. Rev. E, 68 (2003), article 026213.

[20] B. Janiaud, A. Pumir, D. Bensimon, V. Croquette, H. Richter, and L. Kramer, The Eckhaus instability for traveling waves, Phys. D, 55 (1992), pp. 269-286.

[21] T. KAPITULA, Existence and stability of singular heteroclinic orbits for the Ginzburg-Landau equation, Nonlinearity, 9 (1996), pp. 669-685.

[22] T. Kapitula, Stability of weak shocks in $\lambda-\omega$ systems, Indiana Univ. Math. J., 40 (1991), pp. 1193-1219.

[23] T. Kapitula and J. Rubin, Existence and stability of standing hole solutions to complex GinzburgLandau equations, Nonlinearity, 13 (2000), pp. 77-112.

[24] E. Kaplan and V. Steinberg, Phase slippage, nonadiabatic effect, and dynamics of a source of traveling waves, Phys. Rev. Lett., 71 (1993), pp. 3291-3294.

[25] R. Kollár And A. Scheel, Coherent structures generated by inhomogeneities in oscillatory media, SIAM J. Appl. Dyn. Syst., 6 (2007), pp. 236-262.

[26] P. Kolodner, Extended states of nonlinear traveling wave convection. II. Fronts and spatiotemporal defects, Phys. Rev. A, 46 (1992), article 6452.

[27] N. Kopell ANd L. N. Howard, Plane wave solutions to reaction-diffusion equations, Stud. Appl. Math., 52 (1973), pp. 291-328.

[28] Y. Lan, N. Garnier, And P. Cvitanović, Stationary modulated-amplitude waves in the $1 D$ complex Ginzburg-Landau equation, Phys. D, 188 (2004), pp. 193-212.

[29] J. LEGA, Traveling hole solutions of the complex Ginzburg-Landau equation: A review, Phys. D, 152-153 (2001), pp. 269-287.

[30] M. B. Monagan, K. O. Geddes, K. M. Heal, H. Labahn, S. M. Vorkoetter, J. McCarron, And P. DeMarco, Maple Introductory Programming Guide, Maplesoft, Waterloo, ON, 2007; see also http://www.maplesoft.com.

[31] R. Montagne and E. Hernández-García, Localized structures in coupled Ginzburg-Landau equations, Phys. Lett. A, 273 (2000), pp. 239-244.

[32] A. C. Newell, Envelope equations, in Nonlinear Wave Motion, Lectures in Appl. Math. 15, A. C. Newell, ed., American Mathematical Society, Providence, RI, 1974, pp. 157-163.

[33] S. NII, The accumulation of eigenvalues in a stability problem, Phys. D, 142 (2000), pp. 70-86.

[34] K. NozAKi And N. BeKki, Formations of spatial patterns and holes in the generalized Ginzburg-Landau equation, Phys. Lett. A, 110 (1985), pp. 133-135.

[35] L. Pastur, M. T. Westra, D. Snouck, W. van de Water, M. van Hecke, C. Storm, and W. van SAARloos, Sources and holes in a one-dimensional traveling-wave convection experiment, Phys. Rev. E, 67 (2003), article 036305 .

[36] L. Pastur, M. T. Westra, And W. van de Water, Sources and sinks in $1 D$ traveling waves, Phys. D, 174 (2003), pp. 71-83.

[37] J. J. Perraud, A. Dewit, E. Dulos, P. DeKepper, G. Dewel, and P. Borckmans, One-dimensional spirals - Novel asynchronous chemical wave sources, Phys. Rev. Lett., 71 (1993), pp. 1272-1275.

[38] L. Petzold, Automatic selection of methods for solving stiff and nonstiff systems of ordinary differential equations, SIAM J. Sci. Stat. Comput., 4 (1983), pp. 136-148.

[39] F. Plenge, H. Varela, and K. Krischer, Asymmetric target patterns in one-dimensional oscillatory media with genuine nonlocal coupling, Phys. Rev. Lett., 94 (2005), article 198301.

[40] S. Popp, O. Stiller, I. Aranson, And L. Kramer, Hole solutions in the $1 D$ complex Ginzburg-Landau equation, Phys. D, 84 (1995), pp. 398-423.

[41] S. Popp, O. Stiller, I. Aranson, A. Weber, and L. Kramer, Localized hole solutions and spatiotemporal chaos in the $1 D$ complex Ginzburg-Landau equation, Phys. Rev. Lett., 70 (1993), pp. $3880-3883$.

Copyright (C) by SIAM. Unauthorized reproduction of this article is prohibited. 
[42] M. M. Romeo And C. K. R. T. Jones, Stability of neuronal pulses composed of concatenated unstable kinks, Phys. Rev. E, 63 (2001), article 011904.

[43] H. Sakaguchi, Instability of the hole solution in the complex Ginzburg-Landau equation, Progr. Theoret. Phys., 85 (1991), pp. 417-421.

[44] B. Sandstede, Stability of multiple-pulse solutions, Trans. Amer. Math. Soc., 350 (1998), pp. 429-472.

[45] B. Sandstede And A. Scheel, Absolute stability of standing pulses, Nonlinearity, 18 (2005), pp. 331-378.

[46] B. Sandstede And A. Scheel, Defects in oscillatory media: Toward a classification, SIAM J. Appl. Dyn. Syst., 3 (2004), pp. 1-68.

[47] B. Sandstede And A. Scheel, On the stability of periodic travelling waves with large spatial period, J. Differential Equations, 172 (2001), pp. 134-188.

[48] B. Sandstede And A. Scheel, Gluing unstable fronts and backs together can produce stable pulses, Nonlinearity, 13 (2000), pp. 1465-1482.

[49] S. Sasa And T. Iwamoto, Stability of phase-singular solutions to the one-dimensional complex GinzburgLandau equation, Phys. Lett. A, 175 (1993), pp. 289-294.

[50] J. A. Sherratt, A comparison of periodic travelling wave generation by Robin and Dirichlet boundary conditions in oscillatory reaction-diffusion equations, IMA J. Appl. Math., 73 (2008), pp. 759-781.

[51] J. A. Sherratt, Periodic travelling wave selection by Dirichlet boundary conditions in oscillatory reaction-diffusion systems, SIAM J. Appl. Math., 63 (2003), pp. 1520-1538.

[52] J. A. SherratT, On the evolution of periodic plane waves in reaction-diffusion equations of $\lambda-\omega$ type, SIAM J. Appl. Math., 54 (1994), pp. 1374-1385.

[53] M. J. Smith, J. D. M. Rademacher, And J. A. Sherratt, Absolute stability of wavetrains can explain spatiotemporal dynamics in reaction-diffusion systems of lambda-omega type, SIAM J. Appl. Dyn. Syst., 8 (2009), pp. 1136-1159.

[54] M. J. Smith, J. A. Sherratt, And N. J. Armstrong, The effects of obstacle size on periodic travelling waves in oscillatory reaction-diffusion equations, Proc. R. Soc. Lond. A, 464 (2008), pp. 365-390.

[55] O. Stiller, S. Popp, I. Aranson, And L. Kramer, All we know about hole solutions in the CGLE, Phys. D, 87 (1995), pp. 361-370.

[56] M. van HECKE, Coherent and incoherent structures in systems described by the $1 D$ CGLE: Experiments and identification, Phys. D, 174 (2003), pp. 134-151.

[57] M. van HECKE, Building blocks of spatiotemporal intermittency, Phys. Rev. Lett., 80 (1998), pp. 18961899.

[58] M. VAN HECKE AND M. HowARD, Ordered and self-disordered dynamics of holes and defects in the one-dimensional complex Ginzburg-Landau equation, Phys. Rev. Lett., 86 (2001), pp. 2018-2021.

[59] M. van Hecke, C. Storm, And W. van SaArloos, Sources, sinks and wavenumber selection in coupled CGL equations and experimental implications for counter-propagating wave systems, Phys. D, 134 (1999), pp. 1-47.

[60] W. van SaArloos And P. C. Hohenberg, Fronts, pulses, sources and sinks in generalized complex Ginzburg-Landau equations, Phys. D, 56 (1992), pp. 303-367.

Copyright (c) by SIAM. Unauthorized reproduction of this article is prohibited. 